\title{
Points de repère dans l'analyse de la stabilité et de l'interaction génotype-milieu en amélioration des plantes
}

\author{
M Brancourt-Hulmel ${ }^{1 *}$, V Biarnès-Dumoulin ${ }^{1}$, JB Denis ${ }^{2}$ \\ ${ }^{1}$ Laboratoire de génétique et d'amélioration des plantes, Inra, F-80200 Estrées-Mons ; \\ ${ }^{2}$ Laboratoire de biométrie, Inra, route de Saint-Cyr, F-78026 Versailles cedex, France
}

(Reçu le 26 août 1996 ; accepté le 6 mai 1997)

Summary - Guiding marks on stability and genotype-environment interaction analyses in plant breeding. In plant breeding studies, different statistical stabilities between genotypes or genotype-environment interactions (GEI) must often be considered since genotype responses differ from one environment to another. This paper reviews the statistical techniques used in recent literature up to 1996 and the most recent developments are described.

First, stability concepts are reviewed and genotype-environment interaction is defined according to the following notation:

$$
E\left[Y_{g e}\right]=\mu+\alpha_{g}+\beta_{e}+\alpha \beta_{g e}
$$

where $E\left[Y_{g e}\right]$ is the expectation of a given observation $Y_{g e}$ for genotype $g$ and environment $e, \mu$ is the grand mean, $\alpha_{g}$ is the genotype main effect, $\beta_{e}$ the environment main effect and $\alpha \beta_{g e}$ is the interaction between genotype and environment, defined as the complement from the additive model $\left(\mu+\alpha_{g}+\beta_{e}\right)$.

Then, main statistical methods are presented and classified from an interpreting point of view into five main approaches:

(1) Uniparametric approaches: stability or GEI is described with a single parameter. Environmental variance can be set so as to differ for each genotype, which was first introduced by Roemer (1917, cited from Becker and Léon, 1988) and written as follows:

$$
E\left[Y_{g e}\right]=\mu+\alpha_{g} \text { and } V\left[Y_{g e}\right]=\sigma_{g}^{2}
$$

$\mu$ and $\alpha_{g}$ have the same meaning as in the first model and $\sigma^{2}$ are variance parameters associated with each genotype. The joint regression model, first proposed by Yates and Cochran (1938), which uses environment main effect as a pseudo covariate for modelling the interaction term, also belongs to this category:

$$
E\left[Y_{g e}\right]=\mu+\alpha_{g}+\beta_{e}+\rho_{g} \beta_{e}
$$

where $\rho_{g}$ is the genotype slope or genotype regression coefficient that describes the genotype response to environment potentiality estimated by $\beta_{e}$, its main effect. Other terms of the model, $E\left[Y_{g e}\right], \mu$ and $\alpha_{g}$ are defined as in the first model. This family of models is attractive for the simplicity of its interpretations. Most authors have concluded that these models oversimplify and have added new parameters such as goodness of fit. This leads to more sophisticated families of models.

\footnotetext{
* Correspondance et tirés à part

Tél : (33) 03.22.85.75.09; fax : (33) 03.22.85.69.96; courriel : hulmel@ mons.inra.fr
} 
(2) Multiparametric fixed approaches: GEI is modelled by means of several parameters associated with each genotype. There are two basic models: biadditive (or AMMI) models and factorial regression models. They can be extended and combined in several ways, see Gauch (1992) and van Eeuwijk et al (1996).

The multiplicative model is written:

$$
E\left[Y_{g e}\right]=\mu+\alpha_{g}+\beta_{e}+\lambda_{1} \gamma_{g 1} \delta_{e 1}+\lambda_{2} \gamma_{g 2} \delta_{e 2}+\ldots
$$

where $\lambda_{1}$ is the singular value that accounts for the interactive part explained by the first term, $\gamma_{g 1}$ is the normalised genotype vector describing genotype differences and $\delta_{e 1}$ similarly describes the environments; $\lambda_{2}, \gamma_{g 2}$ and $\delta_{e 2}$ are assigned to the second term involving orthogonality constraints with the first term and so on. As previously, other terms of the model, $\left(\mu+\alpha_{g}+\beta_{e}\right)$, correspond to the additive part of the model.

The factorial regression model can be written:

$$
E\left[Y_{g e}\right]=\mu+\alpha_{g}+\beta_{e}+\sum_{h k} G_{g k} \cdot \theta_{k h} \cdot E_{e h}+\sum_{h} \alpha_{g h}^{\prime} \cdot E_{e h}+\sum_{k} \beta_{e k}^{\prime} \cdot G_{g k}
$$

where $\theta_{k h}, \alpha_{g h}^{\prime}$ and $\beta_{e k}^{\prime}$ are regression parameters involving $H$ environment covariates $E_{e h}$ and $K$ genotype covariates $G_{g k}$. Again $\left(\mu+\alpha_{g}+\beta_{e}\right)$ is the additive part of the model.

A common feature of the AMMI model and factorial regression is that both describe the interaction multiplicatively as a genotype score times an environment score. However, in the AMMI model, both parameters are unknown (bilinear model in parameters), while only a single parameter is unknown in regression, implying a linear model. From a practical point of view, regression is thought to be easier for interpretation but on the other hand it requires that relevant covariates be available.

(3) Mixed (random and fixed) parametric approaches: starting from a pioneer work of Shukla (1972), factorial regression models can also be used when environments are considered as a random factor and heteroscedastic genotype variances are introduced; see Denis et al (1997) for a recent development.

(4) Nonparametric approaches: this family includes different methods whose common feature is based on genotype ranking and not on estimation or prediction of genotype performances. This is indeed an attractive aim in many breeding programs where breeders are interested in rank order for choosing the best genotypes. In such cases, relative comparisons are sufficient and there is no need to assess the levels.

(5) Clustering approaches: here the idea is not to obtain a continuous function modelling the interaction but to identify clusters of similar genotypes and/or clusters of similar environments such that most of the interactive variability is captured by the groups of genotypes and/or environments (defining 'between' effects). From a statistical as well as an interpreting point of view, a crucial distinction has to be made according to whether the clusters are determined a priori (by additional information) or a posteriori (based on the data to be explained).

In the last section, comparisons of most of the previous methods are carried out, mainly by means of tables summarising results obtained from the literature (tables II, IV, V, VIII and IX and fig 2). Among them, figure 2 depicts 52 interaction studies using either joint regression, multiplicative approach or factorial regression. These interaction studies are characterised by the proportion of parameters used by the model with respect to the complete interaction (the 'cost' or in the reverse term the 'parsimony') and the proportion of interaction explained by the model (the 'efficiency'). As illustrated in this figure, the AMMI model and factorial regression are equally efficient and much better than joint regression. Our advice is to use factorial regression when relevant covariates are available, owing to its easier interpretation.

\section{stability / genotype-environment interaction / plant breeding}

Résumé - En amélioration des plantes, le chercheur est souvent amené à réaliser des analyses de stabilité ou d'interaction génotype-milieu. Des revues bibliographiques existent sur le sujet et décrivent des approches différentes selon les auteurs. Le présent article propose une classification des principales méthodes utilisées pour une période allant jusqu'en 1996 en mettant l'accent sur les plus récentes, notamment les méthodes qui font intervenir plusieurs paramètres pour décrire la stabilité des génotypes. En vue de comparer la régression conjointe, la modélisation multiplicative de l'interaction (ou modèle AMMI) et la régression factorielle sur la base de l'efficacité (mesurée par le pourcentage de la somme des carrés des écarts de l'interaction décomposée par le modèle) et de la parcimonie (appréciée par le nombre de degrés de liberté utilisés par le modèle), diverses récapitulations ont été réalisées. Pour chaque méthode, elles s'appuient sur la littérature et mentionnent diverses caractéristiques telles que l'espèce étudiée, la variable analysée, le nombre de génotypes et d'environnements, l'efficacité, la parcimonie et le rapport entre les deux dernières. En général, ceci met en valeur la modélisation multiplicative de l'interaction et la régression factorielle. Cette dernière permet en outre de proposer une explication biologique à l'interaction.

stabilité / interaction génotype-milieu / amélioration des plantes 


\section{INTRODUCTION}

L'étude de la stabilité du rendement et de l'interaction génotype-milieu intervient à tous les niveaux de l'amélioration des plantes : le sélectionneur recherche des génotypes présentant une bonne stabilité, tandis que le généticien est souvent confronté de façon involontaire à des problèmes d'analyse de l'interaction. Ceci explique le développement d'un nombre important de modèles pour l'analyse de la stabilité et de l'interaction. Dans ce qui suit, c'est essentiellement la caractérisation des génotypes qui est prise en compte ; pour chaque méthode présentée, la terminologie anglaise est indiquée en italique.

\section{Concepts de stabilité et d'interaction génotype-milieu}

La stabilité du rendement est un critère important pour le choix d'une variété. Parmi la multitude de termes employés, les termes de stabilité phénotypique, de stabilité du rendement et d'adaptation ou d'adaptabilité sont le plus souvent utilisés avec des sens parfois différents. Il n'y a donc pas de définition unique de la stabilité.

La stabilité phénotypique caractérise l'importance des fluctuations du rendement observées pour un même génotype cultivé dans différents milieux. Plus un génotype est stable, moindre est sa variation phénotypique. Les fluctuations observées dépendent des effets du milieu d'une part et de l'existence d'effets d'interaction génotypemilieu d'autre part : les génotypes ne réagissent pas tous de la même façon d'un milieu à un autre. L'interaction est dite «quantitative » si le classement des génotypes se maintient d'un lieu à un autre ; dans le cas contraire, il s'agit d'interaction « qualitative » ou crossover interaction (Romagosa et Fox, 1993 ; Allard et Bradshaw, 1964).

On oppose l'adaptation spécifique ou particulière (specific / narrow adaptation) à une région ou à certains types de milieux à l'adaptation générale (general / wide adaptation) qui désigne un génotype adapté à une large gamme de milieux. On parle aussi de génotypes spécialistes et de génotypes généralistes. D'après Gallais (1992a), l'adaptation est « l'aptitude d'une variété, d'un génotype (au sens large, individu ou groupe d'individus) à bien valoriser des conditions particulières ». L'adaptabilité est « l'aptitude d'un génotype à valoriser des conditions de milieux variés. On parle encore d'adaptation générale ou d'homéostase (homeostasis) ou de stabilité phénotypique ». Le sélectionneur recherchera une adaptation spécifique lorsque le milieu est bien contrôlé, comme en conditions artificielles par exemple, et plutôt une adaptation générale, associée à une adaptation spécifique, lorsque le milieu est plus ou moins contrôlé comme en conditions naturelles (Gallais, 1992a).

Ces définitions rejoignent également les notions de concept dynamique (Becker et Léon, 1988) ou agronomique (Becker, 1981) de la stabilité, selon lequel un génotype est stable si sa réponse au milieu ne présente pas de déviations par rapport à la réponse au milieu de l'ensemble des génotypes étudiés. Ils sont à rapprocher également des différents types de stabilité (2,3 et 4) définis par Lin et al (1986) et Lin et Binns (1988a).

Statistiquement, l'interaction génotype-milieu se définit comme l'écart au modèle additif (Scheffé, 1959). Dans le cadre du modèle additif, pour un génotype $g$ placé dans le milieu $e$, le rendement observé $Y_{g e}$ s'approche en espérance $\left(E\left[Y_{g e}\right]\right)$ par la somme de la moyenne générale $(\mu)$, de l'effet principal du génotype $\left(\alpha_{g}\right)$ et de l'effet principal du milieu $\left(\beta_{e}\right): E\left[Y_{g e}\right]=\mu+\alpha_{g}+$ $\beta_{e}$; alors que le modèle interactif complet prend en plus en compte toute forme possible d'interaction entre les facteurs génotype et milieu : $E\left[Y_{g e}\right]$ $=\mu+\alpha_{g}+\beta_{e}+\alpha \beta_{g e}$ avec $\alpha \beta_{g e}$ interaction entre le génotype $g$ et le milieu $e$. Les différentes définitions de la stabilité correspondent en fait à des méthodes différentes d'analyse, qui modélisent l'effet génotype $\left(\alpha_{g}\right)$ uniquement ou les effets d'interaction génotype-milieu $\left(\alpha \beta_{g e}\right)$ seuls ou encore simultanément les effets $d$ 'interaction génotype-milieu $\left(\alpha \beta_{g e}\right)$, les effets du génotype et du milieu $\left(\alpha_{g}\right.$ et $\beta_{e}$ ) et même parfois la moyenne générale $(\mu)$.

La stabilité est donc une notion plus large que l'interaction et elles sont complémentaires l'une de l'autre. Dans ce qui suit, nous nous placerons du point de vue de l'améliorateur. Si d'un point de vue statistique, il paraît plus logique de dissocier les méthodes qui permettent de mesurer la stabilité de celles qui mettent en œuvre une modélisation de l'interaction ou toute autre modélisation, il nous a paru plus intéressant de les associer pour donner à l'améliorateur des points de repère dans l'analyse de la stabilité et de l'interaction génotype-milieu. Avant de les aborder, nous proposons de traiter l'origine de la stabilité d'un point de vue génétique bien que très peu de références soient disponibles sur ce sujet. 


\section{Origine génétique de la stabilité}

La stabilité selon Gallais (1992a) aurait deux origines : le pouvoir tampon lié à la structure génétique de certains génotypes, à leur hétérogénéité et à leur hétérozygotie d'une part, et l'existence de gènes d'adaptation d'autre part.

Divers travaux ont montré la meilleure stabilité des structures génétiques hétérogènes par des comparaisons entre "population " hétérogène (par exemple une population, un mélange d'hybrides, une variété multilignée, une variété multiclone...) et une "population " homogène (par exemple une lignée parentale, un hybride...). Sur sorgho, les travaux d'Haussmann et Geiger (1994) ont montré la supériorité des mélanges d'hybrides ou des mélanges de lignées par rapport à leurs parents respectifs en culture pure. Sur féverole, Stelling et al (1994) trouvent également une supériorité des structures hétérogènes.

L'effet de la base génétique de l'hybride sur sa stabilité et sa performance est bien connu chez les espèces allogames où la sélection de variétés hybrides se pratique depuis assez longtemps. Chez le maïs par exemple, Eberhart et Russell (1969) ont montré une meilleure stabilité des hybrides doubles par rapport aux hybrides simples. Sur sorgho, Ombakho et Miller (1994) ont observé que les hybrides doubles sont plus stables que les hybrides trois-voies, eux-mêmes plus stables que les hybrides simples.

En ce qui concerne la comparaison entre hybrides et lignées, les résultats sont variables selon les espèces et les auteurs. Chez les espèces allogames, Darwin (cité par Gallais, 1992b) avait déjà signalé que les hybrides F1 de maïs étaient beaucoup plus stables que les lignées. Ceci rejoint les observations de Stelling et al (1994) sur féverole. Tandis que chez le sorgho, les hybrides sont légèrement plus stables que les lignées parentales (Haussmann et Geiger, 1994). Enfin chez les autogames, il semblerait que la meilleure stabilité des hybrides par rapport aux lignées soit controversée. Becker et Léon (1988) ont trouvé cette supériorité sur riz et sur blé ainsi que LefortBuson et Dattée (1985) sur colza tandis que sur pois, des résultats inverses aux précédents ont été trouvés (Sarawat et al, 1994). Enfin Borghi et Perenzi (1990) ont constaté que les hybrides de blé se comportent de la même manière que leurs parents.

Dans les cas où une plus grande stabilité des hybrides par rapport aux lignées est observée, celle-ci est à relier à la manifestation d'hétérosis.
Les hybrides seraient plus stables que leurs parents car plus vigoureux et cette supériorité se manifesterait surtout dans des conditions défavorables : d'après Lefort-Buson et Dattée (1985) sur colza et Giauffret et al (1997) sur maïs. Il en est de même pour l'hybride entre populations chez le dactyle (Breese, 1969).

Globalement, il semblerait donc que l'effet de l'hétérogénéité et de l'hérérozygotie soit variable entre espèces. Ceci est souligné par Stelling et al (1994) qui ont comparé leurs résultats obtenus sur féverole à ceux observés par d'autres auteurs sur trois espèces (colza, maïs et sorgho). Les auteurs expliqueraient cette variation par le système naturel de reproduction distinct entre les espèces citées.

Par ailleurs, la stabilité peut être due à la présence de gènes d'adaptation. La résistance à une maladie affectant le rendement pourra être considérée comme un gène d'adaptation : en cas d'attaque de la maladie dans un milieu donné, le génotype résistant aura un meilleur rendement que le génotype sensible. Tout autre facteur génétique d'adaptation à un milieu peut constituer également un facteur de stabilité tel que la résistance à différents stress, la vigueur au départ, le tallage, la précocité de floraison, le nombre d'épillets par épi, le nanisme... (Hoogendoorn et al, 1988 ; Baril, 1992 ; Nachit et al, 1992a, b ; Worland, 1996). Ceccarelli et al (1992) ont montré chez l'orge que les allèles contrôlant un haut rendement en milieux peu productifs sont au moins partiellement différents de ceux qui contrôlent un haut rendement en milieux productifs. Selon Romagosa et Fox (1993), avant d'établir un programme de sélection, il faut démontrer que les allèles qui contrôlent le rendement dans les deux situations sont différents.

Chez les plantes allogames comme chez les plantes autogames, l'avantage de l'hybride serait dû au fait qu'un génotype, en raison de son hétérozygotie, peut accumuler un plus grand nombre d'allèles favorables dominants contrôlant l'adaptation à différents milieux (Gallais, 1992b). Dans ce cas, l'homeostasie est donc fixable alors qu'elle est impossible à fixer quand elle est due à des gènes allèles codominants situés au même locus (Gallais, 1992b).

\section{Estimations de la stabilité et analyse de l'interaction génotype-milieu}

L'analyse de la stabilité et de l'interaction génotype-milieu a fait l'objet de très nombreuses publi- 
cations en amélioration des plantes. Les principales revues disponibles à ce jour sont, à notre connaissance : Freeman, 1973 ; Denis et Vincourt, 1982 ; Vincourt et al, 1984 ; Lin et al, 1986 ; Westcott, 1986 ; Becker et Léon, 1988 ; Ceccarelli, 1989 ; Crossa, 1990 ; Freeman, 1990 ; Gallais, 1992a, b ; Gauch, 1992 ; Romagosa et Fox, 1993 ; van Eeuwijk, 1996 ; van Eeuwijk et al, 1996 ; Kang et Gauch, 1996. Elles sont complémentaires les unes des autres et intéressantes à consulter pour avoir plus d'informations sur le sujet car elles mettent l'accent sur des méthodes différentes selon les auteurs.

Il existe diverses possibilités pour classer les méthodes permettant l'analyse de la stabilité et de l'interaction. Afin de diriger le lecteur, le présent article propose une classification possible des différentes approches (fig 1). Ainsi plusieurs types seront distingués : les méthodes paramétriques, incluant des modèles à effets fixes et des modèles mixtes (à effets fixes et aléatoires), et les méthodes non-paramétriques. Afin de ne pas compliquer les notations, nous n'avons pas distingué explicitement estimateurs et estimations dans les présentations de méthodes.

Parmi les méthodes paramétriques à effets fixes, on distingue plusieurs catégories selon le nombre de paramètres associés à chaque génotype. Les méthodes uniparamétriques font intervenir un seul paramètre dédié à la stabilité pour décrire les génotypes tandis que les méthodes multiparamétriques en font intervenir plusieurs.
Certaines méthodes uniparamétriques sont des cas particuliers de méthodes multiparamétriques. En revanche, d'autres techniques sont exclusivement uniparamétriques ou multiparamétriques. Au plan statistique, la distinction entre les méthodes uniparamétriques et multiparamétriques ne se justifie donc pas dans tous les cas. Du point de vue de l'analyse de la stabilité, les premières sont des méthodes plus anciennes et mieux connues des améliorateurs tandis que les secondes sont plus récentes, plus complexes et moins largement utilisées pour certaines. C'est pourquoi nous proposons cette distinction et nous ne nous attarderons pas sur les méthodes uniparamétriques qui ont fait l'objet de revues bibliographiques bien complètes afin de détailler davantage les méthodes multiparamétriques.

Enfin, nous traiterons à part des approches fondées sur des modèles fixes lorsque l'améliorateur établit des « groupes » de génotypes et ou de milieux. Les génotypes d'un même groupe sont supposés se comporter de manière similaire suivant les groupes de milieux. Ils présentent donc un même type de stabilité. L'interprétation se réduit alors à celle de la stabilité des différents groupes. Cette démarche revêt un intérêt certain lorsque l'expérimentation est multilocale, pluriannuelle, réalisée selon plusieurs modes culturaux et lorsque de nombreuses variables sont comparées. De plus, la comparaison à des variétés connues ou à des témoins permet une meilleure fiabilité de l'estimation de la stabilité. Nous évoquerons ces méthodes de classification en fin de section.
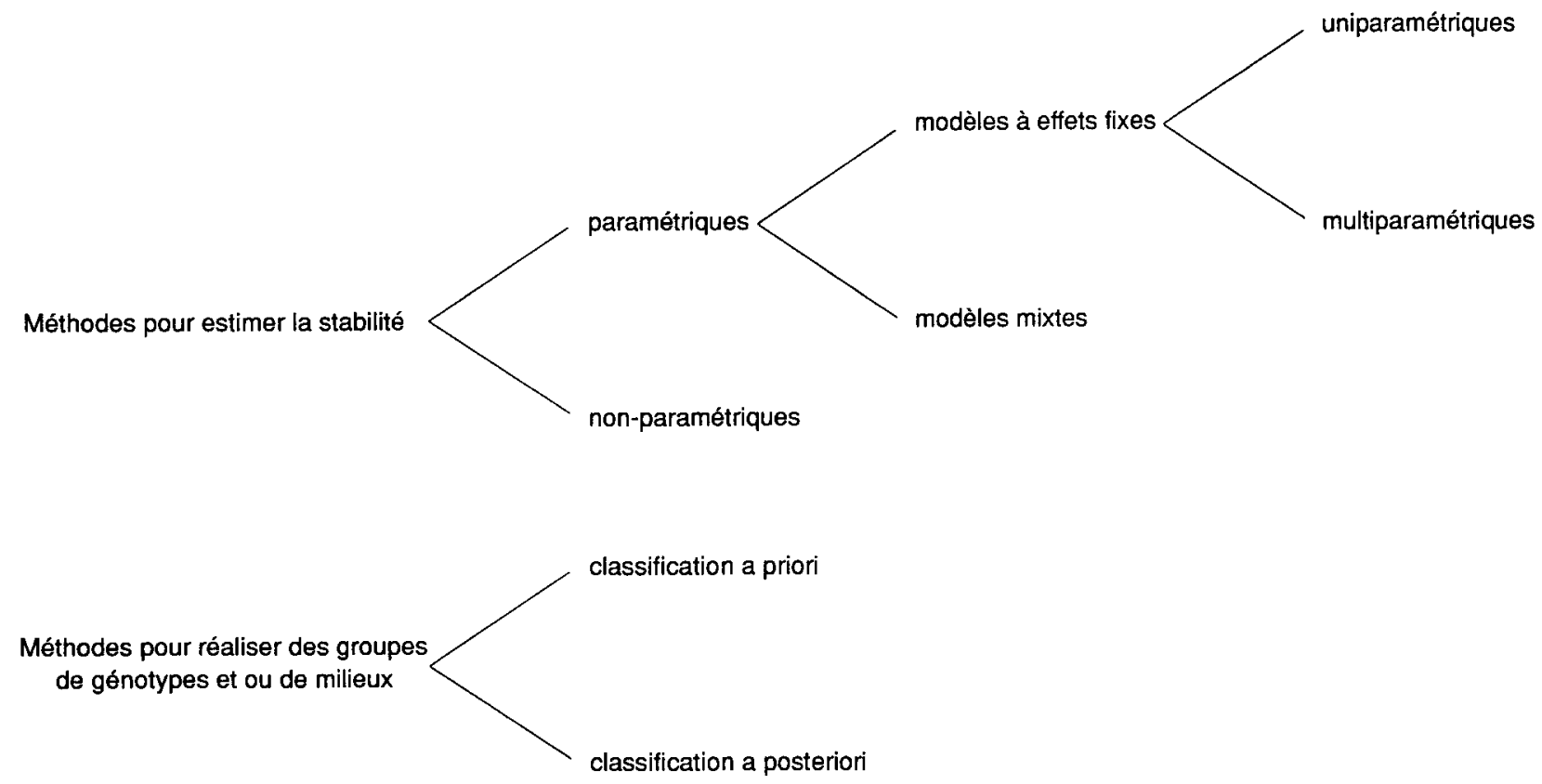

Fig 1. Une classification possible des différentes méthodes d'analyse de la stabilité et de l'interaction.

Fig 1. One possible classification of different methods for the analysis of stability and genotvpe-environment interaction. 


\section{Méthodes pour analyser la stabilité et l'interaction génotype-milieu}

\section{Méthodes uniparamétriques}

Les méthodes uniparamétriques correspondent à des définitions différentes de la stabilité, qui ont été notamment données par Lin et al (1986) et Becker et Léon (1988). Elles comprennent le calcul de paramètres reposant soit sur un modèle prenant en compte uniquement l'effet génotype soit sur un modèle prenant en compte les effets d'interaction génotype-milieu et permettant d'expliciter ces derniers. Lin et al (1986) ont présenté une brève description accompagnée d'équations et de symboles pour la plupart de ces méthodes. Le tableau I en donne un résumé et ces méthodes vont être brièvement décrites dans ce qui suit. Pour plus de détails, nous invitons le lecteur à consulter les revues de Lin et al (1986) et de Becker et Léon (1988).

\section{Modèle prenant en compte uniquement l'effet génotype}

À partir d'un modèle qui prend en compte uniquement l'effet génotype : $E\left[Y_{g e}\right]=\mu+\alpha_{g}$, il est possible de définir des paramètres permettant d'apprécier la stabilité d'un génotype tels que la variance environnementale (environmental variance) ou le coefficient de variation environnementale (coefficient of variability). La variance environnementale se définit de la façon suivante (Roemer, 1917, cité par Becker et Léon, 1988) :

$$
E
$$

$S_{g}^{2}=\sum_{e=1}\left(Y_{g e}-Y_{g .}\right)^{2} /(E-1)$ avec $Y_{g e}$ rendement

du génotype $g$ dans le milieu $e, \mathrm{Y}_{g}$ rendement moyen du génotype $g$ sur tous les milieux et $E$ nombre total de milieux. Le coefficient de variation environnementale est défini ainsi par Francis et Kannenberg (1978): $C V_{g}=S_{g}\left(Y_{g}\right)$. Ces deux paramètres de stabilité relêvent du concept statique défini par Becker et Léon (1988) ou encore du concept biologique développé par Becker (1981), et rejoignent la notion d'homéostasie. Ils ont été classés dans le type I de stabilité par Lin et al (1986).

\section{Modèle prenant en compte les effets d'interaction génotype-milieu}

Différentes approches reposant sur des modèles dérivés du modèle interactif complet, qui s'atta-

Tableau I. Résumé des méthodes uniparamétriques (effets fixes).

Table I. Summary of uniparametric methods (fixed).

\begin{tabular}{|c|c|c|c|}
\hline Méthode & Auteur & $\begin{array}{l}\text { Type selon } \\
\text { Lin et al, } \\
\quad 1986\end{array}$ & $\begin{array}{cc}\text { Concept } & \text { Autre } \\
\text { selon Becker } & \text { définition de } \\
\text { et Léon, } 1988 & \text { la stabilité }\end{array}$ \\
\hline
\end{tabular}

\footnotetext{
Modélisation de l'effet génotype

Variance environnementale $\left(S_{g}^{2}\right)$

Coefficient de variation environnementale $\left(\mathrm{CV}_{\mathrm{g}}\right)$

Modélisation des effets d'interaction génotype-milieu

Écovalence variétale $\left(W_{g}^{2}\right)$

Écovalence environnementale $\left(W_{p}^{2}\right)$

Variance de Shukla $\left(\sigma_{g}^{2}\right)$

Régression conjointe
}

Coefficient de régression $\left(b_{g}\right)$

$$
\left(\rho_{g}\right)
$$

Carré moyen de déviation

à la droite de régression $\left(\mathrm{S}_{d g}^{2}\right)$

Coefficient de détermination $\left(r_{g}^{2}\right)$

Carré moyen de l'effet année hiérarchisé à l'effet lieu $\left(C M_{l / a}\right)$
Roemer 1917, cité par

Becker et Léon, 1988

Francis et Kannenberg, 1978

von Wricke, 1962

Parisot-Baril, 1992

Shukla, 1972

Yates et

Cochran, 1938

Finlay et

Wilkinson, 1963

Eberhart et Russell, 1966

Perkins et Jinks, 1968a

Eberhart et Russell, 1966 ;

Perkins et Jinks, 1968a

Pinthus, 1973 ;

Bilbro et Ray, 1976

Lin et Binns, 1988a statique

homéostasie

1

2

2

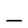

2

statique/ dynamique

adaptation/

statique/

dynamique

dynamique

dynamique

dynamique adaptabilité
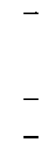
chent à expliciter le terme d'interaction génotype-milieu $\left(\alpha \beta_{g e}\right)$, permettent également de calculer des parametres de stabilité caractérisant les génotypes. Ces méthodes recouvrent le concept dynamique (Becker et Léon, 1988) ou agronomique (Becker, 1981) de la stabilité, selon lequel un génotype est stable si sa réponse au milieu ne présente pas de déviations par rapport à la réponse au milieu de l'ensemble des génotypes étudiés. Elles rejoignent également les notions d'adaptation et d'adaptabilité et les différents types de stabilité (2, 3 et 4) définis par Lin et al (1986) et Lin et Binns (1988a). Parmi ces méthodes, on trouve l'écovalence (ecovalence), la variance de Shukla (Shukla's stability variance), la régression conjointe (joint regression) et ses dérivés.

D'après Lin et al (1986), un génotype présente une stabilité de type 2 s'il réagit parallèlement à la réponse moyenne des génotypes avec lesquels il est testé. Cette stabilité est définie selon la qualité d'ajustement des données au modèle additif ; elle permet de quantifier l'interaction génotype-milieu. Parmi les paramètres statistiques rencontrés pour décrire cette stabilité, on trouve l'écovalence variétale notée $W_{g}^{2}$ (Wricke, 1962). Il s'agit de la contribution du génotype g à la somme totale des carrés des écarts de l'interaction :

$$
W_{g}^{2}=\sum_{e=1}^{E}\left(Y_{g e}-Y_{g .}-Y_{. e}+Y_{. .}\right)^{2}
$$

avec $Y_{g}$, rendement moyen du génotype $g, Y_{\text {.e }}$ rendement moyen du milieu $e$ et $Y$. moyenne générale. De la même façon, on peut définir une écovalence du milieu (Parisot-Baril, 1992). L'estimation d'une variance environnementale sans biais proposée par Shukla (1972) appartient aussi au type 2. Il s'agit d'une combinaison linéaire de l'écovalence. Un génotype stable présente une faible écovalence et une faible variance de Shukla. Ces approches empiriques sont voisines des approches permises par les modèles mixtes qui sont abordées un peu plus loin.

La régression conjointe est l'une des techniques les plus employées pour analyser l'interaction génotype-milieu du rendement chez de nombreuses espèces (tableau II) et aborder les problèmes de stabilité. Plusieurs auteurs ont décrit et élaboré cette méthode : Yates et Cochran (1938), Finlay et Wilkinson (1963), Eberhart et Russell (1966) et Perkins et Jinks (1968a). La démarche des premiers concepteurs ne fut pas basée sur un modèle à ajuster mais constitue plutôt une approche empirique d'explication de l'interaction par une régression simple. Le plus souvent le régresseur est l'effet principal du facteur milieu car il est toujours disponible et facilement interprétable. Cela produit implicitement le modèle suivant :

$$
E\left[Y_{g e}\right]=\mu+\alpha_{\mathrm{g}}+\beta_{\mathrm{e}}+\rho_{\mathrm{g}} \beta_{\mathrm{e}}
$$

avec $\mu$ la moyenne générale, $\alpha_{g}$ l'effet principal du génotype, $\beta_{e}$ l'effet principal du milieu et $\rho_{g}$ le coefficient de régression génotypique (regression coefficient), une mesure de la réponse du génotype à la potentialité du milieu estimée par l'effet principal $\beta_{c}$. En fait, sous cette forme le modèle est bilinéaire en paramètres, c'est une des variantes des modèles biadditifs décrits par Denis et Gower (1996). Si les estimateurs des moindres carrés sont bien connus lorsqu'il n'y pas de données manquantes, c'est la procédure d'estimation en deux étapes, $\left(\mu, \alpha_{\mathrm{g}}\right.$ et $\left.\beta_{\mathrm{e}}\right)$ puis $\left(\rho_{\mathrm{g}}\right)$ sur le terme interactif, qui est la plus souvent utilisée et associée à la dénomination de régression conjointe. Cette pratique se justifie au plan statistique dans la mesure où les effets principaux sont indépendants de l'interaction lorsque les données sont complètes (Milliken et Graybill, 1970).

Les coefficients de régression $\rho_{g}$ renseignent sur l'adaptation ou l'adaptabilité des génotypes et permettent de les classer en trois catégories : les variétés valorisant bien les milieux favorables (coefficient positif), les variétés bien adaptées à des milieux défavorables (coefficient négatif) et les génotypes moyennement adaptés à tous les milieux (coefficient proche de zéro). Finlay et Wilkinson (1963) ont quant à eux considéré les coefficients de régression $b_{g}=1+\rho_{g}$ comme indicateurs de la stabilité des génotypes.

Par ailleurs, différents auteurs ont fort justement proposé de tenir compte aussi de la qualité d'ajustement à la droite de régression conjointe pour mesurer la stabilité des génotypes. Ainsi, Eberhart et Russell (1966) ont suggéré d'utiliser le carré moyen de déviation à la droite de régression ou deviation mean square $\left(s^{2}{ }_{d g}\right)$. Pour Pinthus (1973) et Bilbro et Ray (1976), la stabilité peut être mesurée par le coefficient de détermination ou coefficient of determination $\left(r^{2}\right)$, c'est-àdire le rapport entre la variation expliquée par le modèle et la variation totale, incluant les effets principaux. Ce dernier reflète la proximité des points expérimentaux autour de la droite de régression.

Ainsi, trois paramètres peuvent être employés pour caractériser les génotypes : $\alpha_{g}$, la performance moyenne, $\rho_{g}$, qui traduit la réactivité à la richesse du milieu et $\left(1-r_{g}^{2}\right)$, qui constitue l'estimation d'une variabilité propre à chaque génotype et correspond à la part non linéaire de la réacti- 


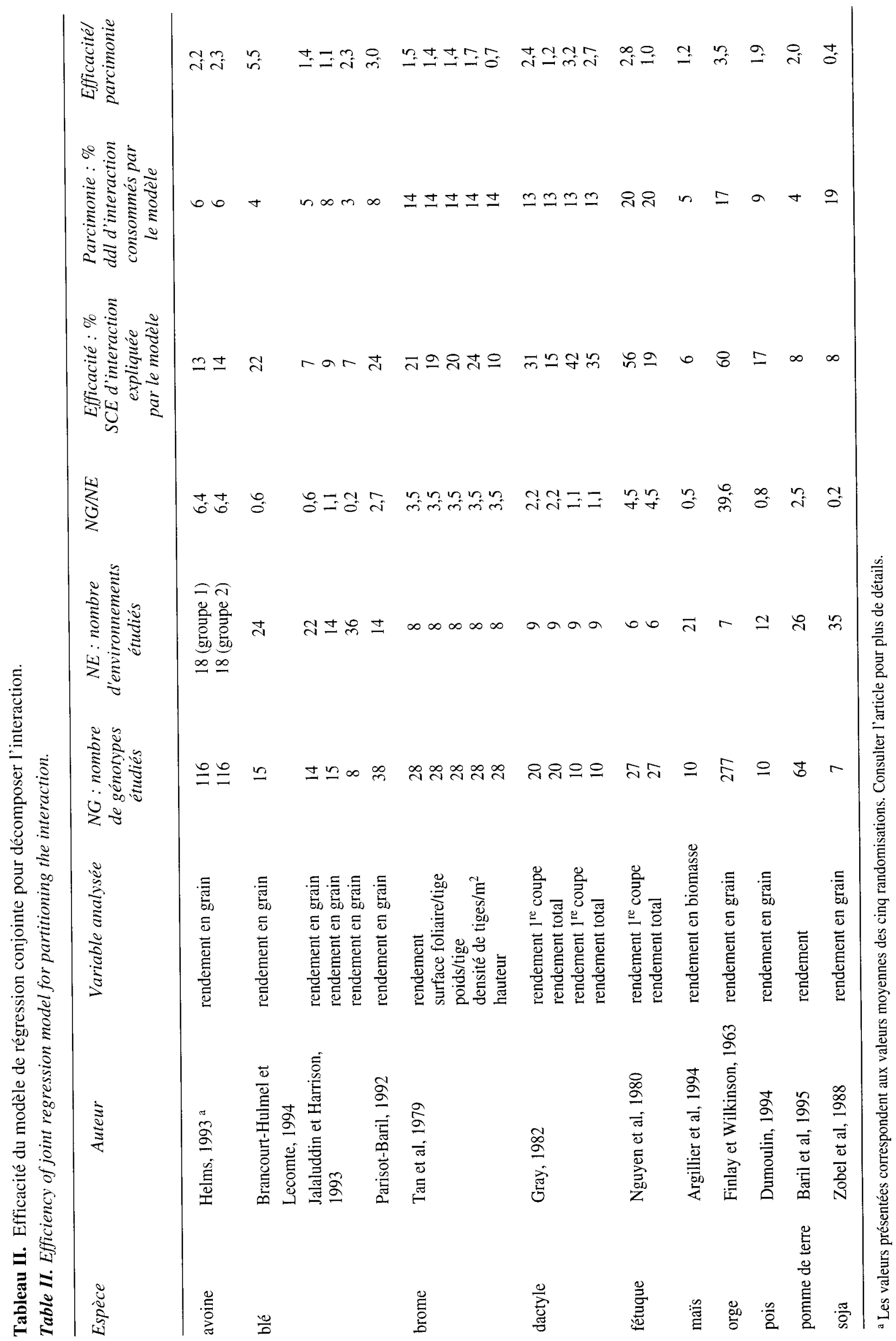


vité des génotypes. Si on considère uniquement la pente pour caractériser la stabilité d'un génotype, la régression conjointe peut être considérée comme une méthode uniparamétrique. Cependant, les autres paramètres sont souvent associés à la pente et cette méthode apparaît donc plus comme un cas particulier des méthodes multiparamétriques.

Quelques compléments ont été apportés à ce modèle. Brown et al (1983) ont proposé la régression sur les moyennes des génotypes. Pour assurer une symétrie entre les deux facteurs, le modèle peut comprendre à la fois la régression sur les moyennes des environnements et la régression sur les moyennes des génotypes (Wright, 1971). Notons aussi que ces effets principaux peuvent être élevés à une certaine puissance (carré, cube,...) ainsi que l'ont proposé Milliken et Graybill (1970). Cette possibilité est offerte par le logiciel Intera (Decoux et Denis, 1991). On parle alors de régression conjointe généralisée (generalized joint regression), où le nombre de paramètres associés aux génotypes est supérieur à 1 . La régression conjointe se classe donc plutôt parmi les méthodes uniparamétriques tandis que la généralisation de cette dernière est de nature multiparamétrique. Gusmao et al (1992) ont préconisé une méthode de régression conjointe « apurée » ou trimmed joint regression : au préalable, les données sont partiellement réestimées, après élimination des données relatives aux génotypes présentant une interaction spécifique. Verma et al (1978) ont proposé de calculer les coefficients de régression sur deux groupes distincts de milieux (respectivement pauvres et favorables) pour détecter les génotypes qui sont à la fois peu réactifs aux milieux pauvres et au contraire très réactifs aux milieux favorables.

La méthode de régression conjointe présente cependant des inconvénients qui ont été énumérés par de nombreux auteurs, notamment Crossa (1990), Gauch (1992) et Romagosa et Fox (1993). Un des points les plus contestés est que l'index environnemental, constitué par les moyennes de l'ensemble des variétés dans chacun des lieux, n'est pas indépendant des données étudiées, ce qui peut introduire un biais dans l'estimation des paramètres de la régression (Freeman, 1973 ; Crossa, 1990 ; Gauch, 1992). En fait, cette méthode est principalement limitée par le faible pourcentage de l'interaction expliquée (tableau II) : il est de l'ordre de $25 \%$ dans la plupart des cas, ceci peut être dû au fait que le modèle consomme peu de degrés de liberté. En revanche, la régression est efficace dans les cas où le nombre de génotypes est très important par rapport au nombre de milieux comme dans l'exemple de Finlay et Wilkinson (1963). Ces mêmes inconvénients se retrouvent pour les méthodes dérivées de la régression conjointe.

Par ailleurs, Lin et Binns (1988a) ont défini un autre paramètre de stabilité, noté type 4 , qui fait intervenir un facteur lié au climat. La variation du milieu est séparée en une composante prévisible due à l'interaction génotype-lieu et une composante imprévisible due à l'interaction génotype-année. Cette dernière permet d'estimer la capacité d'un cultivar à résister à des variations imprévisibles, qui sont en effet causées par les effets années : un génotype stable présente un faible carré moyen de l'effet année hiérarchisé à l'effet lieu $\left(C M_{l / K}\right)$. Avec ce type de paramètre, ainsi qu'avec ceux prenant en compte la qualité d'ajustement à la droite de régression conjointe, la stabilité ou plus exactement l'instabilité est donc mesurée par les irrégularités imprévisibles du génotype dans sa réponse aux environnements.

Pour l'ensemble de ces méthodes, la principale critique réside dans le fait qu'elles ne rendent pas bien compte d'un caractère aussi complexe que la stabilité sur lequel agissent de très nombreux facteurs. Pour lever cet inconvénient, il est nécessaire soit d'en combiner plusieurs, soit d'avoir recours à des analyses multiparamétriques.

\section{Méthodes multiparamétriques}

Les approches présentées précédemment donnent seulement des caractéristiques individuelles de la stabilité mais ne fournissent pas une image globale de la réponse. De plus, un génotype stable selon un des critères ne l'est pas forcément selon les autres. Un seul paramètre ne suffit donc pas pour caractériser la stabilité d'un génotype et il convient d'en associer plusieurs pour une analyse plus complète. Notons que certaines méthodes peuvent appartenir au groupe des méthodes uniparamétriques dans le cas particulier où le nombre de paramètres associés aux génotypes est égal à 1. Les analyses multiparamétriques ont été présentées par divers auteurs (Freeman, 1973 ; Lin et al, 1986 ; Westcott, 1986 ; Becker et Léon, 1988 ; Crossa, 1990 ; Gauch, 1992 ; Romagosa et Fox, 1993) : elles permettent soit une modélisation de l'interaction seule soit une modélisation simultanée des effets d'interaction et des effets principaux génotype et milieu (tableau III).

Par ailleurs, on peut espérer des conclusions plus robustes et générales du fait de la parcimonie (parsimony), appréciée par le nombre de degrés 
Tableau III. Résumé des méthodes multiparamétriques (effets fixes).

Table III. Summary of multiparametric methods (fixed).

Méthode
Modélisation de l'interaction seule
Modèle multiplicatif/modèle AMMI
Régression multiple
Régression factorielle
Modélisation de l'interaction et des effets principaux
Modèle SHMM
Analyse en coordonnées principales
Analyse des coefficients de piste

Auteur

Mandel, 1969, 1971 ; Denis et Gower, 1992, 1996 ;

Gollob, 1968 ; Gauch et Zobel, 1988, 1990, 1992

Hardwick et Wood, 1972 ; Perkins, 1972 ; Wood, 1976 ;

Saeed et Francis, 1984 ; Gorman et al, 1989 ;

Feyerherm et al, 1992

Denis, 1980, 1988

Seyedsadr et Cornelius, 1992 ; Cornelius et al, 1992 ;

Cornelius et al, 1996 ; Crossa et al, 1996

Westcott, 1987

Tai, 1975, 1990 de liberté utilisés par le modèle ou le nombre de paramètres indépendants.

Diverses modélisations de l'interaction sont basées sur plusieurs paramètres de stabilité : le modèle multiplicatif (ou modèle AMMI, additive main effects and multiplicative interaction ou encore modèle biadditif), la régression multiple (multiple regression) et la régression factorielle (factorial regression) que nous allons aborder maintenant. Il existe aussi la régression conjointe généralisée que nous avons mentionnée précédemment. Dans tous ces cas, la stabilité des génotypes n'est pas appréciée par un seul paramètre comme dans le cas de la régression conjointe mais par plusieurs, entraînant une interprétation plus riche au prix de la perte de la simplicité. Par exemple, il n'est en général plus possible de classer les variétés selon un " degré » de stabilité.

Le modèle multiplicatif (ou modélisation multiplicative de l'interaction), proposé par Gollob (1968) et Mandel $(1969,1971)$, permet de décomposer l'interaction en une somme de produits entre des valeurs caractérisant les génotypes et des valeurs caractérisant les milieux. Ce modèle est dénommé AMMI par Gauch (1992) et Gauch et Zobel (1996). Dans le cas complet, l'estimation du modèle d'interaction multiplicative revient à ajuster le modèle additif puis à effectuer la décomposition en valeurs singulières des résidus. Cette technique est aussi utilisée en analyse en composantes principales (principal components analysis), ce qui peut expliquer la façon dont Gauch (1992) définit le modèle. Ce type de modèle appartient à la famille des modèles biadditifs définis par Denis et Gower (1992, 1996). Gauch et Zobel (1988) ont montré qu'il permet une bonne précision «prédictive » dont l'explication statistique invoque "l'effet de Stein » (Gauch, 1990) grâce auquel un sacrifice au niveau du biais peut produire un gain dans la qualité de la prédiction. Autrement dit, mieux vaut un modèle bien estimé et légèrement faux qu'un modèle mal estimé quoique juste. Dans le cas de données manquantes, Gauch et Zobel (1990, cités par Gauch, 1992) ont développé la version EMAMMI (EM pour expectation maximization), qui permet d'estimer ces valeurs à partir des données présentes. Un algorithme des moindres carrés alternés (alternating least squares) est mis en oeuvre dans le logiciel Intera (Decoux et Denis, 1991 ; Denis et Baril, 1992).

Le modèle multiplicatif s'écrit de la façon suivante :

$$
E\left[Y_{g e}\right]=\mu+\alpha_{g}+\beta_{e}+\lambda_{1} \gamma_{g 1} \delta_{e 1}+\lambda_{2} \gamma_{g 2} \delta_{e 2}+\ldots
$$

avec $\mu$ (moyenne générale), $\alpha_{g}$ (effet principal du génotype), $\beta_{e}$ (effet principal du milieu), $\lambda_{1}$ (valeur singulière caractérisant la part de l'interaction expliquée par le terme 1), $\gamma_{\mathrm{g} 1}$ (scores associés aux génotypes précisant les contrastes mis en cause dans le terme 1) et $\delta_{e 1}$ (scores associés aux milieux précisant les contrastes mis en cause dans le terme 1), $\lambda_{2}, \gamma_{g 2}$ et $\delta_{e 2}$ sont respectivement associés au terme 2 et ainsi de suite... Il s'agit donc d'une modélisation de l'interaction par une somme de termes multiplicatifs, chaque terme étant le produit d'une fonction des génotypes $\left(\gamma_{g}\right)$ par une fonction des milieux $\left(\delta_{e}\right)$. À ce modèle est associée la représentation graphique dite du "biplot ", présentée par Kempton (1984) dans le contexte des interactions génotype-milieu. Les génotypes et les milieux sont projetés simultané- 
ment sur un graphique comprenant deux axes correspondant chacun à un terme du modèle (axe 1 en abscisses pour le premier terme et axe 2 en ordonnées pour le second). La stabilité des génotypes est donc décrite par autant de paramètres qu'il y a de termes dans le modèle. Sur le biplot, un point proche de l'origine indique un génotype ou un milieu présentant une faible interaction. Les points qui sont proches représentent des génotypes ou des milieux ayant des comportements similaires ; des points éloignés représentent au contraire des génotypes ou des milieux ayant des comportements très différents. Denis et Gower (1996) proposent de compléter la représentation graphique du biplot par des régions de confiance asymptotiques de formes elliptiques.

Bien qu'il soit difficile de comparer des espèces différentes d'un point de vue pratique, l'observation du tableau IV permet de constater que le modèle multiplicatif explique une bonne part de l'interaction génotype-milieu quelle que soit l'espèce étudiée par les auteurs cités : le premier axe explique généralement plus de $50 \%$ de la somme des carrés des écarts de l'interaction pour environ $30 \%$ de degrés de liberté. Avec deux axes, ces valeurs atteignent respectivement 70 à $100 \%$ et 30 à $50 \%$.

Cette méthode a fait l'objet de publications récentes chez de nombreuses espèces pour analyser le rendement essentiellement : chez le soja (Zobel et al, 1988), le blé (Crossa et al, 1991 ; Nachit et al, 1992a, b), le colza (Shafii et al, 1992), l'orge (Romagosa et al, 1993), le maïs (Cornelius, 1993) et le haricot sec (Saindon et Schaalje, 1993).

Il s'agit plutôt d'une méthode descriptive qui place les génotypes (et les milieux) les uns par rapport aux autres ; parfois des covariables extérieures (relatives aux génotypes ou aux milieux) sont utilisées pour caractériser les directions de variation. Ainsi, certains auteurs ont proposé d'autres méthodes comme la régression multiple pour expliciter l'interaction à l'aide de covariables environnementales (Hardwick et Wood, 1972 ; Perkins, 1972 ; Wood, 1976 ; Saeed et Francis, 1984 ; Gorman et al, 1989 ; Feyerherm et al, 1992). D'autres auteurs ont effectué une régression du rendement moyen des variétés sur les moyennes des milieux estimées à partir de témoins (Freeman et Perkins, 1971 ; Rameau et Denis, 1992) ou par une ou plusieurs variables du milieu (Jones, 1979 ; Nor et Cady, 1979 ; Beckett, 1982 ; Kang et Gorman, 1989 ; Gorman et al, 1989). Les variables environnementales analysées sont essentiellement climatiques avec notamment les températures et les précipitations pendant certaines phases du cycle des plantes, l'humidité relative, et quelques variables édaphiques comme la texture du sol ou encore des techniques culturales telle que la quantité d'azote apportée. Contrairement à la régression conjointe, ces deux procédés permettent de définir un index environnemental indépendant du jeu de données utilisé pour analyser le rendement. De façon symétrique, une variable du génotype peut être utilisée.

La généralisation de cette méthode à plusieurs covariables du milieu et du génotype est la régression factorielle (Denis, 1980, 1988). En 1992, van Eeuwijk a appliqué une méthode dérivée de la régression factorielle, nommée redundancy analysis. Cette dernière intègre également directement des covariables issues de la régression factorielle et enlève les combinaisons de variables « redondantes », d'où son nom. Van Eeuwijk et al (1996) explicitent qu'il s'agit d'une modélisation multiplicative par des combinaisons linéaires de covariables.

Lorsqu'il existe plusieurs covariables associées aux milieux (notées $E_{e h}$ ) et aux génotypes (notées $\left.G_{g k}\right)$, le modèle prend la forme suivante :

$$
\left.E \mid Y_{g e}\right]=\mu+\alpha_{g}+\beta_{e}+\sum_{h k} G_{g k} \cdot \theta_{k h} \cdot E_{l h}+\sum_{h} \alpha_{g h}^{\prime} \cdot E_{l h}+\sum_{k} \beta_{e k}^{\prime} \cdot G_{g k}
$$

où $G_{g k} \cdot \theta_{k l} \cdot E_{e h}$ sont les termes multiplicatifs entre les $\mathrm{H}^{\prime}$ covariables du milieu et les $\mathrm{K}$ covariables du génotype, $\alpha_{g h}^{\prime}$ est le coefficient de régression génotypique résiduel sur les covariables du milieu $E_{e h}$ et $\beta$, ${ }_{e k}$ est le coefficient de régression résiduel du milieu sur les covariables du génotype $G_{g k}$. Cette méthode a l'avantage de tester directement les covariables susceptibles d'influencer l'interaction contrairement au modèle multiplicatif et aux modèles associés. Elle peut être complétée par l'analyse de l'effet des covariables sur les effets principaux. Lorsqu'il existe une seule covariable du milieu et une seule covariable du génotype, l'écriture du modèle devient :

$$
E\left[Y_{g e}\right]=\mu+\alpha_{g}+\beta_{e}+G_{g} \cdot \theta \cdot E_{\mathrm{e}}+\alpha_{g}^{\prime} \cdot E_{e}+\beta_{e}^{\prime} \cdot G_{k}
$$

Des études récentes (depuis 1992) portant sur différentes espèces ont fait appel à la régression factorielle et ont montré son efficacité pour analyser l'interaction (tableau V). Cette approche, appliquée au rendement dans tous les exemples cités, explique entre 50 et $70 \%$ de la somme des carrés des écarts de l'interaction pour un pourcentage de degrés de libertés compris entre 20 et 40 . L'utilisation de covariables du milieu semble être plus fréquente que celle de covariables génotypiques. Pour certaines espèces, peut-être est-il 


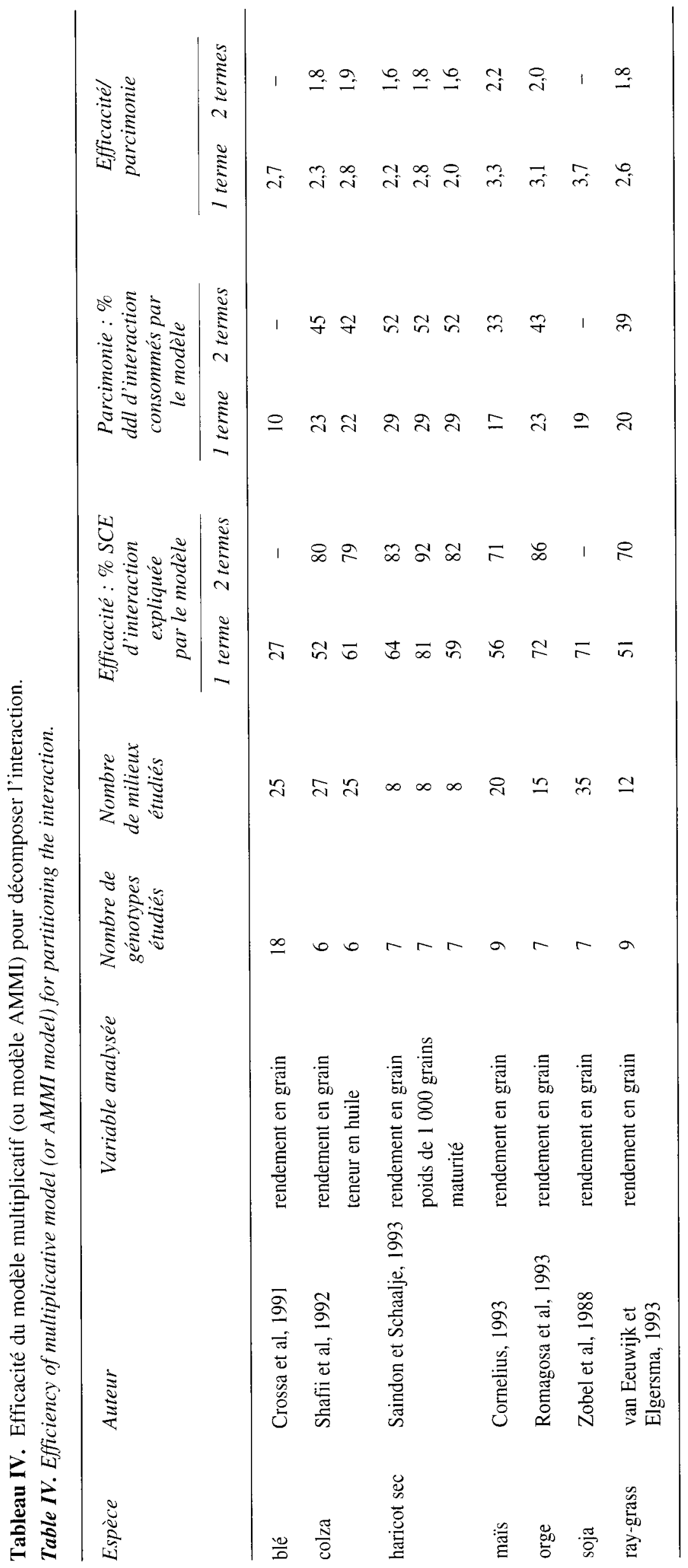




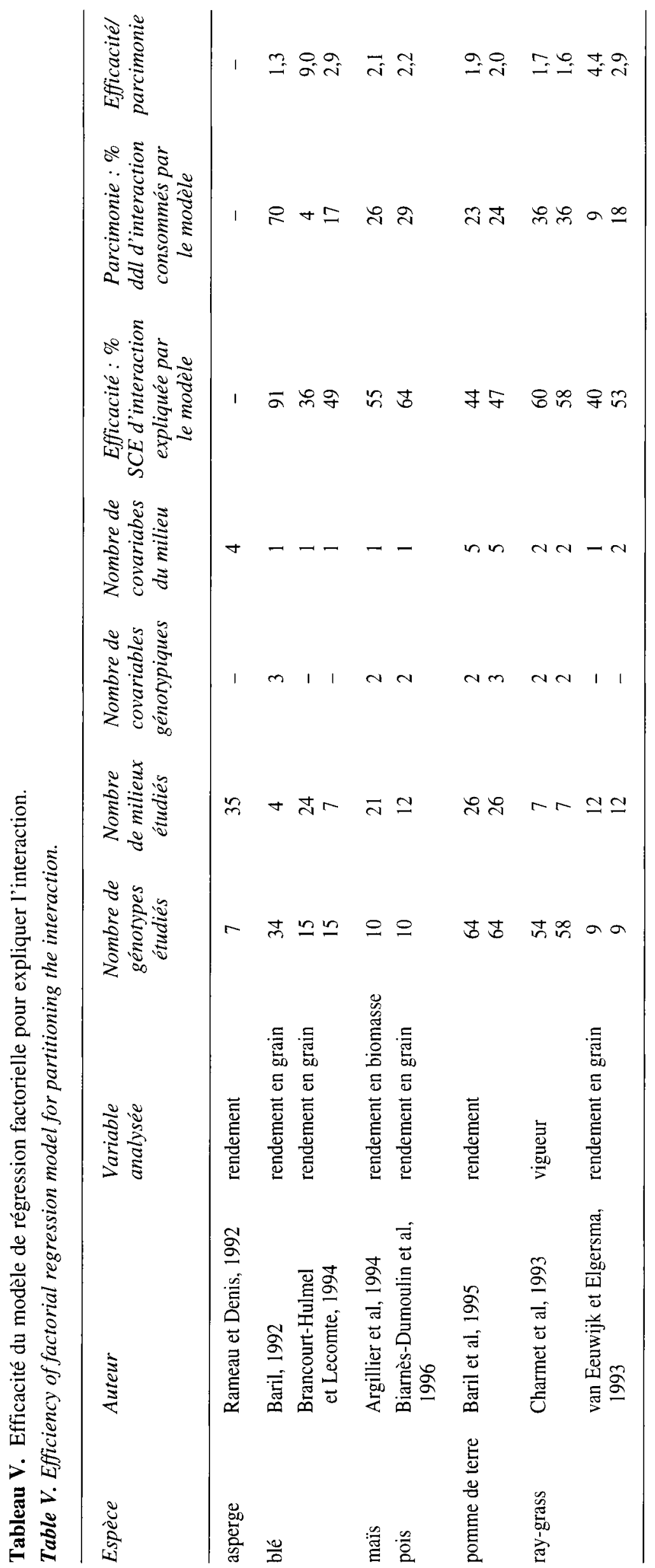


plus immédiat de disposer de covariables intéressantes sur les milieux que sur les génotypes. Une autre raison est liée aux objectifs de l'interprétation statistique : si on veut prédire les performances relatives de deux génotypes dans un milieu non expérimenté, il suffit de connaître les valeurs des covariables pour ce nouveau milieu, les effets principaux disparaissant dans la différence. Parmi les variables climatiques étudiées, on trouve le bilan hydrique (Charmet et al, 1993 ; Biarnès-Dumoulin et al, 1996), les températures souvent associées aux précipitations (Rameau et Denis, 1992 ; Charmet et al, 1993 ; van Eeuwijk et Elgersma, 1993). De nombreuses autres variables du milieu, qui ne concernent pas uniquement le climat mais aussi les données édaphiques (Baril et al, 1995), les pratiques culturales, les pressions parasitaires (Baril, 1992) ont également été analysées. Parmi les covariables génotypiques testées, la précocité de floraison semble un facteur important pour expliquer l'interaction génotype-milieu (Argillier et al, 1994 ; Biarnès-Dumoulin et al, 1996). Pour Baril (1992), le poids de mille grains, la sensibilité à la verse et la longueur de l'épi ont permis d'expliquer $91 \%$ de l'interaction chez le blé tendre d'hiver. Cependant, il semble difficile d'étudier ces variables sans connaître les dates de réalisation des différents stades de la culture et sans faire intervenir les diverses composantes du rendement, d'où la nécessité de réaliser un diagnostic agronomique préliminaire (Brancourt-Hulmel et Lecomte, 1994).

Outre les analyses modélisant l'interaction uniquement, il en existe d'autres qui décomposent simultanément l'interaction et les effets principaux. Il s'agit notamment du modèle SHMM, de l'analyse en coordonnées principales et de l'analyse des coefficients de piste, que nous allons aborder maintenant.

Le modèle SHMM, que l'on pourrait traduire par modélisation multiplicative globale translatée (shifted multiplicative model), développé par Seyedsadr et Cornelius (1992) et Cornelius et al (1992), est une variante des modèles biadditifs (Cornelius et al, 1996 ; Crossa et al, 1996). Il s'agit en effet d'une décomposition multiplicative, que les auteurs appellent analyse en composantes principales modifiée, dans la mesure où l'estimation $\mu$, qui correspond à la moyenne générale, est remplacée par une autre estimation, qui permet de diminuer davantage la résiduelle. Ce modèle prend sa signification autour des mots " shifted " et " multiplicative ", "shifted " indiquant qu'on a « déplacé » la moyenne et «multiplicative » indiquant la présence de termes multi- plicatifs dans l'écriture du modèle. Les deux effets principaux classiques ne sont pas pris en compte.

Le modèle SHMM s'écrit de la façon suivante :

$$
E\left[Y_{g e}\right]=\mu^{\prime}+\lambda^{\prime}, \zeta_{g 1} \cdot \eta_{e 1}+\lambda^{\prime}{ }_{2} \cdot \zeta_{g 2} \eta_{e 2}+\ldots
$$

avec $\mu^{\prime}$ une constante, $\lambda^{\prime}$, valeur singulière pour le terme $1, \zeta_{g 1}$ scores associés aux génotypes pour le terme $1, \eta_{\ell 1}$ scores associés aux milieux pour le terme $1, \lambda_{2}, \zeta_{g 2}$ et $\eta_{e 2}$ sont respectivement associés au terme 2 et ainsi de suite... Cette équation est identique à celle du modèle multiplicatif, à la disparition près des deux effets principaux; leur part d'explication est donc reportée dans la série des termes multiplicatifs. Bien que ce modèle ait été utilisé avec succès dans certains cas (Jackson et al, 1993, 1994), il présente les deux principaux inconvénients suivants : il ne prend pas en compte les effets additifs du génotype et du milieu et l'interprétation de ses paramètres est rendue délicate par sa non-orthogonalité intrinsèque. Par exemple, l'estimation du premier terme varie suivant le nombre de termes muliplicatifs retenus dans le modèle.

Quant à l'analyse en coordonnées principales ou principal coordinate analysis, il s'agit d'une généralisation de l'analyse en composantes principales, qui utilise toute mesure de similarité entre individus autre que la distance euclidienne (Euclidean distance). Westcott (1987) a proposé une telle méthode, qu'il a illustrée à partir de onze variétés de maïs du Cimmyt expérimentées dans 31 lieux en 1979. Il a utilisé la distance suivante pour mesurer la similarité entre les génotypes dans un environnement donné : $d_{g g^{\circ}}=$ $\left[\max -\left(Y_{g}+Y_{g}\right) / 2\right] /($ max-min $)$ avec max désignant le rendement moyen maximal obtenu par un génotype dans l'environnement considéré, $\min$ le rendement moyen minimal, $Y_{g}$, le rendement moyen du génotype $g$ dans le milieu et $Y_{g^{\prime}}$, le rendement moyen du génotype $g^{\prime}$ dans ce même milieu. C'est une méthode descriptive où chaque génotype est décrit par plusieurs coordonnées appelées coordonnées principales (principal coordinates), calculées de façon à reproduire au mieux les distances $d_{g g^{\prime}}$.

Enfin, l'analyse des coefficients de piste ou path coefficient analysis est une forme d'analyse multivariable. Elle diffère de l'analyse en composantes principales par la construction du modèle, qui peut être obtenu sur la base du développement chronologique séquentiel d'une série de composantes du rendement ou « modèle causal » (Tai, 1975, 1990). Le rendement peut s'exprimer, en première approximation, comme la combinaison linéaire de différentes variables ou composantes 
(par exemple, nombre d'épis par $\mathrm{m}^{2}$, nombre de grains par épi et poids d'un grain pour le blé ou nombre de tiges par $\mathrm{m}^{2}$, nombre de tubercules par tige et poids d'un tubercule pour la pomme de terre). Ensuite, on peut rechercher l'influence directe des ressources du milieu pendant la phase de formation de ces composantes et obtenir ainsi une nouvelle combinaison linéaire de « variables causales ", qui permettent de renseigner sur le comportement des génotypes pendant les différentes phases.

L'équation suivante constitue un exemple d'écriture du modèle d'analyse des coefficients de piste :

$$
E\left[Y_{g e}\right]=\mu_{\mathrm{g}}+v_{1 \mathrm{~g}} \cdot r_{1 e}+v_{2 g} \cdot r_{2 e}+v_{3 g} \cdot r_{3 e}
$$

avec $\mu_{g}$ le rendement moyen du génotype $g, v_{1 g}$, $v_{2 g}$ et $v_{3 g}$ les coefficients mesurant la réponse du génotype aux effets de l'environnement pendant les 3 phases d'élaboration du rendement et $r_{1 e}, r_{2 e}$ et $r_{3 e}$, les composantes indépendantes qui sont déterminées par une procédure de carrés moyens (Tai, 1990) et qui pondèrent comme en régression multiple les effets du milieu pendant ces mêmes phases. Une telle démarche a été appliquée sur blé dur dans des environnements limités en eau (Simane et al, 1993) et en 1994 par Tai et al sur pomme de terre.

\section{Modèles mixtes}

Les modèles présentés jusqu'à présent s'inscrivent dans le cadre des modèles à effets fixes, c'est-à-dire que la variance des observations ne dépend que d'un seul paramètre, $\sigma^{2}$. Bien que moins répandues, d'autres modélisations ont été proposées dans le cadre de modèles mixtes (mixed models). Un des auteurs les plus anciens à avoir préconisé ce type d'approche est sans doute Shukla (1972). Une revue des généralisations possibles est réalisée par Denis et al (1997).

Le modèle s'écrit :

$$
E\left[Y_{g e}\right]=\mu+\alpha_{g}+\mathrm{B}_{e}+\rho^{\prime}{ }_{g} \cdot z_{e}+\mathrm{AB}_{g e}
$$

où $z_{e}$ est une covariable environnementale connue, le facteur environnement (e) est considéré comme aléatoire et l'interaction, $\mathrm{AB}_{g e}$, est supposée dépendre du génotype $: \mathrm{V}\left(\mathrm{AB}_{g e}\right) \stackrel{g e}{=} \sigma_{g}^{2}$.

$\mathrm{Ce}$ modèle permet donc de considérer au moins un des facteurs comme aléatoire. Par exemple, l'améliorateur considère les milieux dans lesquels il teste ses cultivars en cours de sélection, comme représentatifs de la population de milieux qu'il vise. Ou encore, le généticien étudie les caractéristiques de sa population de génotypes pour quelques milieux contrastés. Bien entendu, le nombre de niveaux du facteur considéré comme aléatoire doit être en rapport avec l'estimation des composantes de la variance.

Ce modèle permet en outre d'associer une modélisation de l'interaction entre les deux facteurs (fixe et aléatoire) au moyen de régressions $\left(\rho^{\prime}{ }_{g} z_{e}\right)$, ou de termes multiplicatifs. Des fonctions simples des composantes de la variance peuvent alors être associées au concept de stabilité. Un développement sur ces modèles de régression factorielle se trouve dans van Eeuwijk et al (1996) ; la modélisation multiplicative mixte a été proposée par Oman (1991). Enfin, cette modélisation offre une variance différente pour chacun des génotypes (hétéroscédasticité), attribuant explicitement un paramètre $\left(\sigma_{g}^{2}\right)$ pour décrire la stabilité de chaque génotype. Il existe d'autres approches qui prennent en compte l'hétéroscédasticité et qui sont mentionnées par Denis et al (1997).

Les modèles de ce genre, actuellement en plein essor grâce aux possibilités nouvelles des logiciels statistiques, paraissent attractifs par leur souplesse mais présentent l'inconvénient d'être exigeants quant à la quantité et la répartition des données nécessaires. Beaucoup de travail reste encore à faire au niveau de leur traitement statistique.

\section{Méthodes non-paramétriques}

Toutes les approches précédentes se définissent à partir de paramètres, d'autres sont non-paramétriques et constituent une alternative aux premières (Hühn, 1996 ; voir aussi tableau VI). La plupart sont basées sur le rang des génotypes dans chaque environnement, ce sont alors à la fois les effets du génotype et les effets d'interaction génotype-milieu qui sont pris en compte. Il faut corriger au préalable les données en leur retranchant l'effet génotype si l'on veut que les rangs ne reflètent plus que les effets d'interaction génotype-milieu (Hühn et al, 1993).

Ces approches détectent uniquement les inversions de classement (crossover interactions) et confondent les interactions quantitatives (noncrossover interactions) avec l'absence d'interaction. De plus, même en cas d'inversion des classements, l'ordre de grandeur des différences n'est pas pris en compte, leur intérêt en est par conséquent diminué. Pour l'analyse des interactions qualitatives, elles présentent toutefois plusieurs avantages par rapport aux méthodes paramétriques : la réduction voire l'élimination de biais causé par les valeurs extrêmes ou les erreurs de 
Tableau VI. Résumé des méthodes non-paramétriques.

Table VI. Summary of nonparametric methods.

Méthode

Auteur

Concept selon

Becker et Léon, 1988

Moyenne des rangs centrés $\left(S_{g(1)}\right)$

Hühn, 1990a, b

dynamique

Variance des rangs $\left(S_{g(2)}\right)$

Hühn, 1990a, b

dynamique

Somme des différences de rangs rapportées

Hühn, 1990a, b

dynamique

au rang moyen du génotype $\left(S_{g(3)}\right)$

Somme des rangs

(rendement + variance de stabilité de Shukla)

Kang, 1988 ; Kang et Pham, 1991

Indice de fiabilité

Eskridge et Mumm, 1992 ;

Eskridge et al, 1993

Méthode combinatoire

Guénoche et al, 1994

mesures, l'absence d'hypothèses sur la distribution des observations, l'homogénéité des variances et l'additivité des effets ne sont pas nécessaires. De plus, elles sont facilement utilisables et interprétables.

Hühn (1990a) a proposé trois mesures nonparamétriques (nonparametric measures) qui combinent le rendement et la stabilité. Ces statistiques $\left(S_{g(1)}, S_{g(2)}\right.$ et $\left.S_{g(3)}\right)$, basées sur les rangs des génotypes dans chaque environnement, s'expriment de la façon suivante :

$$
\begin{aligned}
S_{g(1)}= & \frac{\sum \mid e^{e}}{\frac{E(E-1)}{2}} \\
S_{g(2)}= & \frac{\sum\left(r_{g e}-r_{g .}\right)^{2}}{E-1}-r_{g e^{\prime}} \mid \\
S_{g(3)}= & \frac{e}{r_{g .}}
\end{aligned}
$$

où $r_{g e}$ est le rang du génotype $g$ dans le milieu $e$, $r_{g e}$, est le rang du même génotype dans le milieu $e^{g}, r_{g}$ est la moyenne des rangs du génotype $g$ dans tous les milieux et $E$ le nombre total de milieux. Sous l'hypothèse nulle d'additivité, Hühn (1996) a montré que ces mesures pouvaient être comparées à des distributions de $\chi^{2}$ pour proposer des tests sur le comportement des génotypes. Appliquant ces trois mesures à un essai officiel d'inscription de blé tendre d'hiver, il a vérifié qu'elles sont presque parfaitement liées lorsque les données sont corrigées par l'effet du génotype étudié (Hühn, 1990b).

Kang (1988) a développé aussi une méthode qui considère à la fois le rendement et la stabilité : il attribue le premier rang au génotype le plus productif et, de la même façon, le premier rang au génotype qui possède la variance de Shukla la plus faible. Pour chaque génotype, les deux rangs sont additionnés et le meilleur génotype est celui qui possède la somme la plus faible (indice 1). Cette méthode donne le même poids aux rangs associés au rendement et à la stabilité. De façon identique, Kang et Pham (1991) ont décrit d'autres indices, qui donnent plus de poids au rendement, de deux fois (pour l'indice 2) jusqu'à cinq fois (pour l'indice 5). Ceux-ci semblent peu intéressants car ils induisent une sélection des génotypes sur le niveau de rendement essentiellement. L'indice 1 (somme des rangs de Kang, 1988) semble donc préférable. Ces auteurs indiquent également que l'écovalence peut remplacer la variance de Shukla.

Par ailleurs, Eskridge et Mumm (1992) et Eskridge et al (1993) ont décrit un autre indice mesurant l'importance de la stabilité qu'ils nomment « fiabilité » ou reliability, estimation basée sur la probabilité que le génotype étudié dépasse les performances d'un témoin. Plus récemment, Guénoche et al (1994) ont présenté une méthode combinatoire pour sélectionner des variétés dans un réseau d'essais. Cette méthode est empruntée 
aux procédures utilisées en théorie du choix social. Elle est basée sur l'établissement d'ordres pour toutes les variétés dans chaque milieu et la comparaison des variétés deux à deux. Pour chaque couple de variétés g et g' dans un milieu donné, la meilleure des deux est définie. Puis pour l'ensemble des milieux, on détermine le nombre de fois (ou de milieux) où la variété g est supérieure à la variété g'. Les auteurs ont illustré cette méthode à l'aide d'un essai multilocal de colza très incomplet et ont considéré uniquement le rendement bien que cette procédure puisse prendre en compte plusieurs caractères différents.

Comme pour toutes les approches examinées dans cet article, ces mesures non-paramétriques de stabilité sont dépendantes des nombres mais aussi des ensembles de génotypes et de milieux considérés. Il nous semble cependant que la réserve doit être d'autant plus forte à leur endroit que l'information retenue est fort réduite : des rangs de performances et non plus des performances. De plus, la question des pondérations entre le rendement et la stabilité n'est pas exclusive des méthodes non-paramétriques et se place dans le cadre très général de la sélection sur index multicaractère non considéré dans cet article.

\section{Méthodes pour réaliser des groupes de génotypes et/ou de milieux}

Un dernier type d'analyses concerne les méthodes de classification. Lin et al (1986) et Westcott (1986) ont répertorié les principales méthodes de classification auxquelles il convient d'ajouter un certain nombre de propositions plus récentes indiquées dans le tableau VII.

On distingue les classifications a priori qui font intervenir des informations extérieures et les classifications a posteriori qui s'appliquent aux données elles-mêmes, faute de mieux.

La plupart des méthodes de classification reposent sur un index de dissimilarité basé sur un calcul de distance ou sur l'ajustement à un modèle ou encore sur l'utilisation de coefficients de corrélation. De plus, cet index se définit soit à partir d'un seul critère ou bien de plusieurs.

\section{Classification a priori}

Une première approche fait intervenir une information extérieure pour réaliser des groupes a priori. Denis (1979) suggère par exemple de clas-

Tableau VII. Résumé des méthodes de classification.

Table VII. Summary of clustering methods.

Classification a priori

Classification a posteriori

Méthode basée sur un seul critère

Index basé sur un calcul de distance Distance euclidienne

\section{Distance euclidienne réduite}

Distance généralisée de Mahalanobis

Index basé sur l'ajustement à un modèle /régression conjointe (partie linéaire) /régression conjointe (partie non linéaire) /modèle SHMM

Utilisation de coefficients de corrélation

Utilisation du carré moyen d'interaction

Méthode basée sur plusieurs critères
Denis, 1979 ; Imrie et al, 1981 ; Brown et al, 1983 ;

Parisot-Baril, 1992 ; Balfourier et Charmet, 1991 ; Peterson, 1992
Hanson, 1970 ; Mungomery et al, 1974 ; Johnson, 1977 ;

Ghaderi et al, 1980

Abou-El-Fittouh et al, 1969 ; Byth et al, 1976 ;

Fox et Rosielle, 1982 ; Fox et al, 1990

Ghaderi et al, 1982 ; Weatherup, 1994

Lin et Thompson, 1975

Perkins et Jinks, 1968b

Cornelius et al, 1993 ; Crossa et al, 1993

Habgood, 1977 ; Krenzer et al, 1992 ; Ghaderi et al, 1982 ; Campbell et Lafever, 1977 ; Yau et al, 1991

Byth et al, 1976 ; Denis, 1979 ; Lin, 1982 ; Ramey et Rosielle, 1983 ; Corsten et Denis, 1990 ; Lin et Butler, 1990

Lefkovitch, 1985 ; Lin et Binns, 1991 ; Lin et al, 1992 ; Cooper et al, 1994 
ser les variétés et les milieux selon leur origine géographique. Ainsi, Parisot-Baril (1992) a pu expliquer chez le blé tendre d'hiver une bonne partie de la variation due à l'interaction en regroupant les génotypes étudiés en fonction de leur origine de sélection et les milieux en fonction du lieu d'expérimentation. Imrie et al (1981) ont également obtenu de bons résultats chez le soja vert (Vigna radiata) en regroupant les génotypes expérimentés en fonction de leur date de floraison. Enfin, Brown et al (1983) ont utilisé des variables environnementales (température, photopériode) pour classer les milieux. Balfourier et Charmet (1991) ont choisi quant à eux l'analyse factorielle des correspondances (correspondence analysis) pour étudier les relations entre caractères agronomiques et facteurs écogéographiques chez des populations françaises de ray-grass anglais tandis que Peterson (1992) a opté pour l'analyse factorielle (factor analysis) sur blé tendre d'hiver où les milieux ont été regroupés par zones de production.

\section{Classification a posteriori}

\section{Méthodes basées sur un seul critère}

La première méthode est basée sur la détermination d'un index de dissimilarité ou de dissemblance basé sur un calcul de distances pour réaliser les groupes. Mungomery et al (1974) et Johnson (1977) ont utilisé la distance euclidienne pour établir des groupes de génotypes. La distance euclidienne entre deux génotypes $g$ et $g$ ' est définie par : $\quad d_{g g^{\prime}}^{2}=\sum_{e=1}^{E}\left(y_{g e}-y_{g^{\prime} e}\right)^{2} \quad$ où $Y_{g e}$ représente la

performance du génotype $g$ dans l'environnement $e, Y_{g}{ }{ }$ désigne la performance du génotype $g$ ' dans l'environnement $e$ et $E$ correspond au nombre d'environnements étudiés. Géométriquement, chaque génotype peut être représenté par un point dont les coordonnées correspondent aux performances de ce génotype dans différents milieux représentés par les axes d'un espace. Les génotypes sont classés en fonction de leur proximité dans cet espace. La distance euclidienne a également été proposée par Hanson (1970) comme mesure de la stabilité des génotypes. Elle peut aussi permettre de regrouper les milieux (Ghaderi et al, 1980). Abou-El-Fittouh et al (1969), Byth et al (1976), Fox et Rosielle (1982) et Fox et al (1990) ont quant à eux fait appel à la distance euclidienne réduite ou standardisée. Enfin, Ghaderi et al (1982) et Weatherup (1994) ont utilisé la distance généralisée de Mahalanobis
(Mahalanobis distance), qui est équivalente à la distance euclidienne réduite lorsque les moyennes ne sont pas corrélées.

Une autre approche consiste à établir un index à partir de l'ajustement à un modèle décomposant l'interaction génotype-milieu. Lin et Thompson (1975) l'ont calculé à partir de l'ajustement à un modèle de régression conjointe (Finlay et Wilkinson, 1963) en regroupant les génotypes présentant des ordonnées à l'origine et des pentes similaires. Cette méthode nécessite que la déviation par rapport au modèle de régression conjointe soit faible par rapport à l'erreur. Pour lever cet inconvénient, Perkins et Jinks (1968b) ont examiné la partie non linéaire de l'ajustement. La méthode proposée par Cornelius et al (1993) et Crossa et al (1993) est basée sur l'ajustement à un modèle, le SHMM (shifted multiplicative model). La « distance » est estimée à partir des résidus à ce modèle à un terme multiplicatif. Mais ceci nécessite également un bon ajustement du modèle aux données.

Par ailleurs, l'utilisation de coefficients de corrélation (correlation coefficients) permet de repérer les génotypes ayant un comportement identique (ou au contraire différent) face aux variations du milieu (Habgood, 1977 ; Krenzer et al, 1992). Cette méthode permet également de réaliser une classification des génotypes (Ghaderi et al, 1982) ou des milieux (Campbell et Lafever, 1977 ; Yau et al, 1991) : les deux génotypes (ou milieux) les plus fortement corrélés sont regroupés en premier ; les autres genotypes (ou milieux) sont ensuite ajoutés successivement jusqu'à ce que l'ensemble des génotypes (ou milieux) ne forme plus qu'un seul groupe.

En outre, Lin (1982) et Ramey et Rosielle (1983) ont proposé d'effectuer les regroupements en se basant sur le carré moyen de l'interaction. Alors que le procédé proposé par Lin (1982) consiste à minimiser le carré moyen d'interaction pour chaque nouveau groupe constitué, la méthode HASS (hierarchical agglomerative sum of squares) de Ramey et Rosielle (1983) minimise le carré moyen d'interaction pour l'ensemble des groupes constitués à chaque étape de la classification hiérarchique. Lin et Butler (1990) ont utilisé la méthode de Lin (1982). La biclassification proposée par Corsten et Denis (1990) est basée sur l'interaction également. En outre, les effets moyens du génotype et du milieu peuvent s'ajouter au terme d'interaction dans le calcul des distances. Cette possibilité est offerte par Intera (Decoux et Denis, 1991). Le principe est de regrouper les génotypes et les milieux de façon à 
ce que l'interaction soit faible à l'intérieur des groupes et forte entre les groupes. Cette méthode rejoint celle préconisée par Byth et al (1976) et Denis (1979).

\section{Méthodes basées sur plusieurs critères}

D'autres modes de classification font appel à une approche multicritère. Lin et al (1992) ont fait intervenir des moyennes génotypiques, des coefficients de régression et des déviations à la régression tandis que Lefkovitch (1985) a défini la dissimilarité entre génotypes par trois mesures simultanées : le rendement moyen sur tous les milieux, la variance environnementale et la réponse des génotypes aux variations du milieu (ou pattern). Lin et Binns (1991) et Lin et al (1992) ont mis au point plusieurs méthodes de classification multicritères et des programmes associés qui font intervenir les ordonnées à l'origine et les pentes des régressions conjointes (ou de régressions faisant intervenir un index du milieu), l'écovalence de Wricke (1962), la variance définie par Shukla (1972), la mesure de la supériorité d'un génotype (Lin et Binns, 1988b) et le paramètre de stabilité de type 4 (Lin et Binns, 1988a). Cooper et al (1994), après avoir utilisé la distance euclidienne pour établir les groupes, ont vérifié leur cohérence chez le blé par des mesures de corrélation et ont analysé le lien avec les composantes du rendement et la production de matière sèche.

Dans toutes ces démarches de classification, le point le plus délicat concerne la détermination $d u$ nombre de groupes de génotypes et de milieux. Tandis que de nombreux auteurs ont fixé ce dernier de façon arbitraire (Mungomery et al, 1974 ; Byth et al, 1976 ; Shorter et al, 1977 ; Ghaderi et al, 1982 ; Brown et al, 1983 ; Brandle et Arthur, 1992), d'autres ont proposé des méthodes graphiques (Lin et Butler, 1988 ; Parisot-Baril, 1992) ou statistiques (Corsten et Denis, 1990 ; Lin et Morrison, 1992 ; Baril et al, 1994) voire des seuils de pourcentage de somme de carrés d'écarts de l'interaction expliquée (Baril et al, 1994). Bull et al (1994) ont proposé de considérer les blocs d'un essai comme des milieux différents, et ils déterminent le nombre de groupes lorsque les blocs d'un même essai se regroupent, cela revient à utiliser l'erreur résiduelle (interaction génotype-bloc) des essais individuels comme référence. De façon similaire, Bull et al (1992) ont proposé d'utiliser le regroupement de témoins.

\section{Comparaison des différentes méthodes. Importance des méthodes multiparamétriques}

\section{Méthodes uniparamétriques : comparaison, répétabilité et héritabilité des paramètres}

Les relations existant entre les différents paramètres de stabilité présentés précédemment ont été analysées par divers auteurs (Becker, 1981 ; Pham et Kang, 1988 ; Piepho et Lolito, 1992 ; Jalaluddin et Harrison, 1993). Quelle que soit l'espèce étudiée par ces auteurs, on distingue généralement deux groupes de paramètres à l'intérieur desquels de fortes corrélations existent :

- le coefficient de régression $\left(b_{g}\right)$ de Finlay et Wilkinson (1963), la variance environnementale $\left(S_{\mathrm{p}}^{2}\right)$ et le coefficient de variation environnementale $\left(C V_{g}\right)$ qui caractérisent l'adaptation au milieu, d'une part ;

- l'écovalence $\left(W_{g}^{2}\right)$ de Wricke (1962), la variance de stabilité $\left(\sigma_{g}^{2}\right)$ de Shukla (1972), le carré moyen de déviation à la régression $\left(\mathrm{s}_{d g}^{2}\right)$ de Eberhart et Russell (1966) et le coefficient de détermination $\left(r_{g}^{2}\right)$ de Pinthus (1973), qui correspondent à la stabilité intrinsèque des génotypes, d'autre part.

Pour des corrélations faisant intervenir d'autres paramètres, le tableau VIII nous montre des valeurs plus nuancées et parfois inversées.

La répétabilité de certains de ces paramètres, c'est-à-dire la corrélation observée entre les mesures d'un même paramètre dans des milieux différents, a également été analysée par plusieurs auteurs (Eagles et Frey, 1977 ; Pham et Kang, 1988 ; Lin et Binns, 1991 ; Jalaluddin et Harrison, 1993 ; Helms, 1993) chez de nombreuses espèces (tableau IX). Selon les deux derniers auteurs, les mesures de stabilité seraient dans l'ensemble assez peu répétables, sauf peut-être pour $b_{g}$. Selon Eagles et Frey (1977), qui ont étudié 80 lignées d'avoine dans 24 milieux, $\sigma_{g}^{2}$ serait répétable pour le rendement en paille mais pas pour le rendement en grain. Léon et Becker (1988) ont par ailleurs observé une certaine répétabilité pour cinq mesures de stabilité $\left(S_{g}^{2}, b_{g}, s_{d g}^{2}, r_{g}^{2}\right.$ et $\left.W_{g}^{2}\right)$ en comparant les résultats obtenus sur une année à ceux relatifs à plusieurs années. Les coefficients de corrélation sont moyens (autour de 0,5 ) et significatifs pour $S_{g}^{2}$ et $b_{g}$. Ils sont en revanche faibles et très variables selon les expérimentations pour les trois autres paramètres. Tous ces résultats ne se retrouvent pas chez d'autres espèces comme le montre le tableau IX. Il est même possible de rencontrer des résultats contradictoires pour une 
Tableau VIII. Corrélations entre différents paramètres issus de méthodes uniparamétriques et non-paramétriques. Table VIII. Correlations between different parameters obtained from uniparametric and nonparametric methods.

\begin{tabular}{|c|c|c|c|c|c|}
\hline $\begin{array}{l}\text { Paramètres } \\
\text { considérés }\end{array}$ & $\begin{array}{c}\text { Valeurs } \\
\text { des corrélations }\end{array}$ & Auteur & $\begin{array}{l}\text { Paramètres } \\
\text { considérés }\end{array}$ & $\begin{array}{c}\text { Valeurs } \\
\text { des corrélations }\end{array}$ & Auteur \\
\hline \multirow[t]{3}{*}{$b_{g}-S_{g}^{2}$} & $0,73^{*} / 0,88^{*}$ & (1) & $S_{g}^{2}-\sigma_{g}^{2}$ & $-0,09 / 0,53^{*}$ & (3) \\
\hline & $0,91^{*}$ & (2) & $S_{g}^{g}-r_{g^{g}}^{r^{2}}$ & 0,50 & (2) \\
\hline & $0,92 * / 0,99 *$ & (3) & $S_{g}^{2}-S_{g(1)}^{0}$ & $-0,13 / 0,40^{*}$ & (1) \\
\hline \multirow[t]{2}{*}{$b_{g}-C V_{g}$} & $0,81^{*}$ & (2) & $S_{g}^{2}-S_{g(2)}$ & $-0,21 / 0,35^{*}$ & (1) \\
\hline & $-0,03 / 0,89^{*}$ & (3) & $s_{d g}^{2^{g}}-\stackrel{C}{C} V_{g}$ & $-0,35 / 0,52^{*}$ & (2) \\
\hline$b_{g}-W_{g}^{2}$ & $-0,27 * / 0,27$ & (1) & $s_{d g}^{2}-W_{g}^{2^{3}}$ & $0,90^{*} / 0,96^{*}$ & (1) \\
\hline \multirow{2}{*}{$b_{g}-s_{d g}^{2}$} & $-0,27^{*} / 0,21$ & (1) & $s_{d g}^{2}-\sigma_{g}^{2}$ & $0,96^{*}$ & (2) \\
\hline & $-0,24 / 0,39$ & (3) & & & \\
\hline \multirow{2}{*}{$b_{g}-\sigma_{g}^{2}$} & $-0,18$ & (2) & & $0,91 * / 0,97 *$ & (3) \\
\hline & $-0,31 / 0,43$ & (3) & & & \\
\hline$b_{g}-r_{g}^{2}$ & $0,73^{*}$ & (2) & $s_{d g}^{2}-r_{g}^{2}$ & $-0,64^{*}$ & (2) \\
\hline$\dot{b_{g}}-S_{g(1)}$ & $-0,27^{*} / 0,19$ & (1) & $s_{d g}^{2}-S_{g(1)}$ & $0,64 * / 0,82 *$ & (1) \\
\hline$b_{g}-S_{g(2)}$ & $-0,24 * / 0,24$ & (1) & $s_{d g}^{2}-S_{g(2)}$ & $0,71 * / 0,89 *$ & (1) \\
\hline \multirow{2}{*}{$S_{g}^{g}-C V_{g}$} & $0,83^{*}$ & (2) & $\sigma_{g}^{2}-C V_{g}$ & 0,07 & (2) \\
\hline & $0,10 / 0,96^{*}$ & (3) & & $-0,45 / 0,63^{*}$ & (3) \\
\hline$S_{g}^{2}-W_{g}^{2}$ & $-0,23 * / 0,43^{*}$ & (1) & $C V_{g}-r_{g}^{2}$ & 0,46 & (2) \\
\hline \multirow[t]{2}{*}{$S_{g}^{2}-s_{d g}^{2}$} & $-0,22 / 0,53^{*}$ & (1) & $W_{g}^{2}-S_{g(\mathbf{1})}$ & $0,71 * / 0,85^{*}$ & (1) \\
\hline & $-0,04 / 0,49^{*}$ & (3) & $W_{g}^{2}-S_{g(2)}$ & $0,79 * / 0,91 *$ & (1) \\
\hline$S_{g}^{2}-\sigma_{g}^{2}$ & 0,08 & (2) & $S_{g(1)}-S_{g(2)}$ & $0,87^{*} / 0,95^{*}$ & (1) \\
\hline
\end{tabular}

Sur une même ligne sont indiquées les corrélations extrêmes rencontrées par les auteurs ; ${ }^{*}$ significatif au seuil de $5 \% ;(1)=$ Piepho et Lolito, $1992 ;(2)$ = Jalaluddin et Harrison, $1993 ;(3)$ = Pham et Kang, 1988.

Tableau IX. Répétabilité des différents paramètres issus de méthodes uniparamétriques.

Table IX. Repeatability of different parameters obtained from uniparametric methods.

\begin{tabular}{|c|c|c|c|c|}
\hline Paramètre & Avoine & Blé & Maïs & Orge \\
\hline$b_{g}$ & $0,57 * / 0,64 *(2)$ & $-0,73 * / 0,81 *(4)$ & - & - \\
\hline$S_{g}^{2}$ & $0,89 *(3)$ & $0,05 / 0,48(4)$ & $\begin{array}{l}-0,47 / 0,49(5) \\
0,12 / 0,47^{*}(5)\end{array}$ & $0,85^{*}(3)$ \\
\hline$C V_{g}$ & - & $-0,07 / 0,88^{*}(4)$ & $\begin{array}{l}-0,15 / 0,52(5) \\
-0,10 / 0,40(5)\end{array}$ & - \\
\hline$W_{g}^{2}$ & $0,74 *(3)$ & - & - & $0,60(3)$ \\
\hline$s_{d g}^{2}$ & $\begin{array}{c}0,12 / 0,25^{*}(1) \\
0,06 / 0,23^{*}(2) \\
0,50(3)\end{array}$ & $-0,10 / 0,30(4)$ & - & $0,34(3)$ \\
\hline$\sigma_{g}^{2}$ & $\begin{array}{c}0,06 / 0,10(1) \\
0,23^{*} / 0,35^{*}(2)\end{array}$ & $-0,54 / 0,31$ & $\begin{array}{l}-0,15 / 0,32(5) \\
-0,17 / 0,34(5)\end{array}$ & - \\
\hline$r_{g}^{2}$ & - & $-0,45 / 0,83^{*}(4)$ & - & - \\
\hline$C M_{I / a}$ & $0,76^{*}(3)$ & - & - & $0,83 *(3)$ \\
\hline
\end{tabular}

Sur une même ligne sont indiquées les corrélations extrêmes rencontrées par les auteurs ; * significatif au seuil de $5 \% ;(1)=$ Eagles et Frey, $1977 ;(2)=$ Helms, $1993 ;(3)=$ Lin et Binns, $1991 ;(4)=$ Jalaluddin et Harrison, 1993 ; (5) = Pham et Kang, 1988. 
rencontrer des résultats contradictoires pour une espèce donnée comme pour le blé par exemple.

En outre, Lin et Binns (1991) ont mesuré l'héritabilité de quatre de ces paramètres pour différentes céréales à paille et ont trouvé dans leurs essais que $S_{g}^{2}$ et le paramètre de stabilité de type 4 de Lin et Binns (1988a) $C M_{1 / a}$ sont héritables tandis que $W_{g}^{2}$ et $s_{d g}^{2}$ ne le sont pas. Sur trèfle, Kearsey et al (1987) ont montré que le coefficient de régression $b_{g}$ est héritable. Seuls les deux premiers paramètres et le coefficient de régression pourraient donc être utilisés en sélection.

\section{Comparaison des méthodes non-paramétriques}

Kang et Pham (1991) ont comparé différentes méthodes non-paramétriques sur maïs à l'aide de cinq essais de variétés-populations : deux indices de Hühn, $S_{g(3)}$ (Hühn, 1990a) et un indice antérieurement défini (Hühn, 1979, cité par Kang et Pham, 1991) et les cinq indices de Kang (1988). Il existe des variations selon les essais : tantôt les indices de Kang aboutissent à la sélection de plus d'individus que ceux de Hühn, tantôt c'est l'inverse. Les deux auteurs proposent donc d'utiliser simultanément les deux types d'indices en sélection. Mais du fait qu'il y a une perte d'information par le passage aux rangs, il n'est pas évident que l'utilisation des seules méthodes nonparamétriques soit totalement efficace. De plus, dans la pratique, le nombre d'individus sélectionnés est contingenté par des contraintes expérimentales car le sélectionneur est limité par le nombre de parcelles qu'il peut mettre en place, ce qui l'amène à réaliser le meilleur compromis.

\section{Comparaison des méthodes uniparamétriques et non-paramétriques}

Hühn (1990b) a comparé les statistiques nonparamétriques qu'il a définies avec des mesures uniparamétriques de la stabilité. Elles sont fortement corrélées avec l'écovalence et les déviations à la régression linéaire tandis qu'elles sont faiblement corrélées avec le coefficient de régression et la variance environnementale. Piepho et Lolito (1992), quant à eux, ont obtenu des résultats assez comparables et ont estimé que $S_{\mathrm{g}(1)}$ et $S_{g(2)}$ peuvent être utilisées comme des alternatives à $W_{g}^{2}$ et à $\sigma_{g}^{2}$. Par ailleurs, Léon et Becker (1988) ont observé que la répétabilité du paramètre $S_{g(2)}$ sur une année était comparable à celle estimée pour cinq mesures paramétriques $\left(S_{\mathrm{g}}^{2}, b_{g}, s_{d g}^{2}, r_{g}^{2}\right.$ et $\left.W_{g}^{2}\right)$.

Eskridge et al (1992) ont montré que la « fiabilité » est reliée à plusieurs autres paramètres de stabilité $: b_{g}, s_{d g}^{2}$ et $\sigma_{g}^{2}$. Cependant, les estimations de la «fiabilité » seraient plus répétables que ces derniers et constitueraient donc de meilleures mesures des caractéristiques génétiques des cultivars.

\section{Comparaison des méthodes uniparamétriques et multiparamétriques}

Parmi les méthodes décrites précédemment, les plus étudiées sont celles qui décomposent l'interaction. Pour comparer celles-ci à partir de données expérimentales, il faut tenir compte d'une part, de l'efficacité (efficiency), appréciée par le pourcentage de la somme des carrés des écarts de l'interaction expliquée par le modèle, et d'autre part, de la parcimonie, appréciée par le nombre de degrés de liberté utilisés. Un modèle optimal présente le meilleur rapport entre l'efficacité et la parcimonie et peut se définir comme un modèle qui décompose la plus grande partie de l'interaction avec un faible nombre de degrés de liberté et qui en outre permet d'en comprendre les bases biologiques ou agronomiques.

Peu d'exemples de comparaison de méthodes existent à ce jour. Quelques auteurs pourtant en ont utilisé plusieurs et les ont comparées sur la base de leur efficacité à expliquer l'interaction (tableaux II, IV et V). Trois méthodes sont principalement concernées : la régression conjointe, le modèle multiplicatif et la régression factorielle.

La régression conjointe apparaît bien moins efficace que les deux autres méthodes pour décomposer l'interaction. En effet, la régression conjointe correspond à un modèle de régression factorielle à une covariable, qui est le rendement moyen des milieux. Ceci peut donc expliquer que la régression factorielle à plusieurs covariables soit généralement plus puissante pour analyser l'interaction génotype-milieu que la régression conjointe (Argillier et al, 1994 ; BrancourtHulmel et Lecomte, 1994 ; Dumoulin, 1994 ; Baril et al, 1995). On peut par ailleurs considérer que les modèles multiplicatifs constituent une généralisation de la régression conjointe (Cruz, 1992). Ceux-ci sont donc également plus performants pour expliquer l'interaction génotypemilieu que la régression conjointe (Argillier et al, 1994 ; Baril et al, 1995).

La régression factorielle donne des résultats comparables à ceux obtenus avec le modèle multiplicatif : les deux techniques ont en effet permis de sélectionner les mêmes variables du milieu pour expliquer l'interaction chez le ray-grass (van 
Eeuwijk et Elgersma, 1993). Ces deux méthodes ont par ailleurs permis d'expliquer une part comparable de l'interaction chez la pomme de terre (Baril et al, 1995) avec des nombres de degrés de liberté relativement proches. Chez le maïs, Argillier et al (1994) ont également trouvé des résultats similaires. L'efficacité et la parcimonie semblent donc équivalentes pour les deux modèles. Cependant, la régression factorielle paraît plus intéressante car elle suggère une interprétation biologique des interactions génotypemilieu et permet d'en comprendre les bases biologiques ou agronomiques. De plus, le modèle statistique est linéaire et non pas bilinéaire, il permet donc des procédures d'inférence plus précises (par exemple pour effectuer des tests ou calculer des intervalles de confiance).

La figure 2, qui regroupe les données des tableaux II, IV et V, montre la relation entre l'efficacité et la parcimonie observées pour les trois méthodes. Il faut préciser que, contrairement aux études précédentes, ces dernières ont été appliquées pour la plupart à des lots de données différents. Pour la régression conjointe, on peut constater une grande variabilité pour le pourcentage de la somme des carrés des écarts de l'interaction décomposée par le modèle (la majorité des points se situant entre 0 et $30 \%$ ) et pour le pourcentage de degrés de liberté consommés par le modèle ( 0 à $20 \%$ ). La figure met en évidence que la régression factorielle et le modèle multiplicatif sont nettement plus efficaces que la régression conjointe bien que moins parcimonieux. Pour ces deux modèles, la part d'interaction expliquée est d'autant plus grande que le nombre de degrés de liberté augmente. Il semble que, pour le modèle multiplicatif, le premier axe (AMMII) apporte le meilleur compromis entre l'efficacité et la parcimonie : en effet, un deuxième axe (AMMI2) consomme beaucoup de degrés de liberté alors qu'il ne décompose guère plus l'interaction.

Toutes les méthodes présentées possèdent des avantages et des inconvénients. Mais elles ne sont pas forcément exclusives et peuvent être employées simultanément dans des modélisations « hybrides » en fonction du cas étudié. Ainsi, on peut combiner une classification sur les génotypes avec l'usage de covariables associées aux environnements (Denis, 1992). Un ajustement multiplicatif successif à une régression factorielle peut également être effectué avec le logiciel Intera (Decoux et Denis, 1991). L'utilisation de termes multiplicatifs comme covariables en régression factorielle peut aussi donner des résultats intéressants (Baril, 1992). De nombreuses autres combinaisons de méthodes sont possibles ; leur mise en ouvre ne dépend que du choix effectué par les utilisateurs.

\section{CONCLUSION}

Des méthodes très diverses existent pour analyser la stabilité et l'interaction génotype-milieu.

Parmi les méthodes uniparamétriques, la régression conjointe est la plus utilisée car elle est facile à mettre en ouvre et est assez aisée à interpréter. Elle semblerait convenir à des analyses faisant intervenir un grand nombre de génotypes

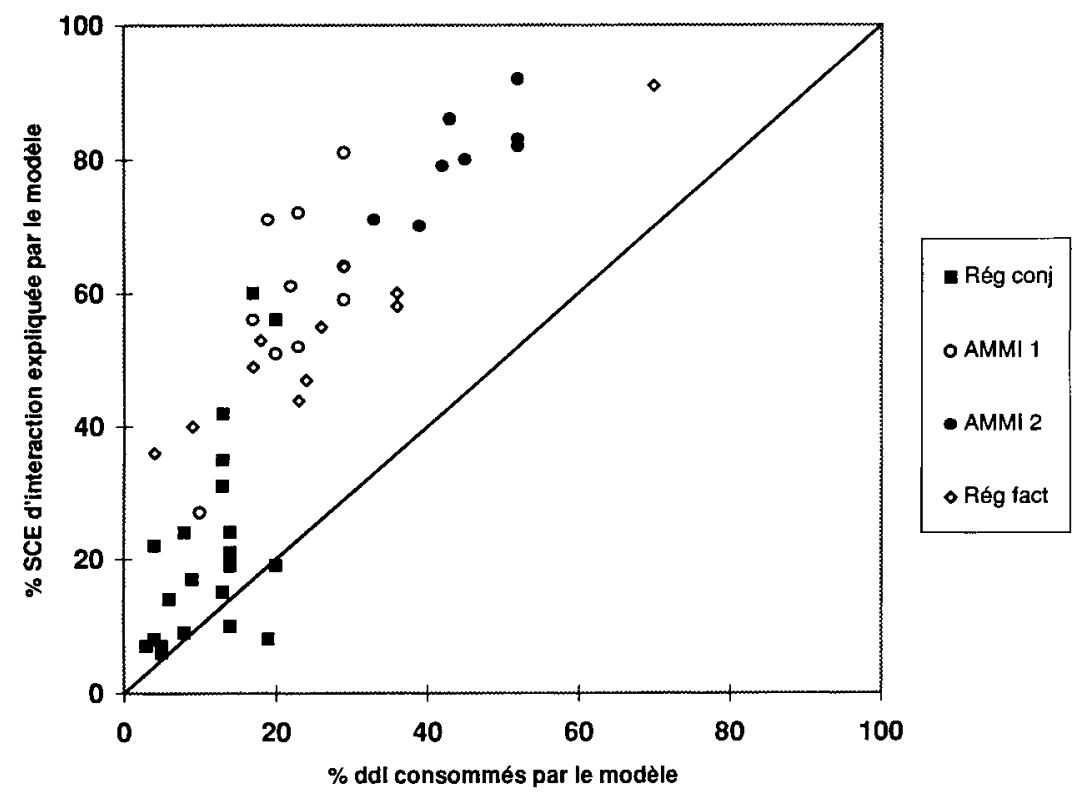

Fig 2. Comparaison de trois modèles : régression conjointe, modèle multiplicatif (ou modèle AMMI) et régression factorielle.

Fig 2. Comparison of three models: joint regression, multiplicative model (or AMMI model) and factorial regression. 
variés dans un nombre limité de milieux, comme dans le cas de l'étude de collections. Mais elle ne tient pas compte du caractère multidimensionnel du comportement des génotypes. Selon l'espèce, l'améliorateur peut choisir d'utiliser certaines de ces méthodes uniparamétriques en fonction des groupes de corrélation auxquels elles appartiennent d'une part, et de leur caractère répétable et héritable d'autre part.

Les méthodes multiparamétriques semblent plus adaptées pour décrire la réponse des génotypes à différentes conditions de milieux. Ces techniques statistiques, qui reposent sur des calculs complexes, n'ont pu se développer qu'avec l'apparition d'ordinateurs puissants et sont donc relativement récentes. Parmi ces dernières, le modèle multiplicatif et la régression factorielle permettent dans la plupart des cas de décomposer une part importante de l'interaction : ces deux méthodes présentent en général une efficacité et une parcimonie comparables. Cependant, la régression factorielle paraît plus complète. En effet, elle ne fournit pas seulement une description des données mais elle permet aussi d'intégrer directement des informations relatives aux milieux ou aux génotypes et de donner une signification biologique aux phénomènes d'interaction génotype-milieu.

Quant aux méthodes non-paramétriques, elles sont simples à mettre en œuvre mais ne permettent d'appréhender que les interactions qualitatives.

Les différentes approches présentées comportent toutes des avantages et des inconvénients. Mais ce ne sont que des pièces d'un jeu de construction qui peuvent s'assembler les unes avec les autres. Il peut être intéressant d'en utiliser plusieurs pour avoir une information plus complète sur la stabilité des génotypes et sur les effets d'interaction génotype-milieu. De plus, parallèlement à toutes ces analyses, l'améliorateur peut être amené à établir des groupes de génotypes et ou de milieux à l'aide de méthodes de classification a priori ou a posteriori. Ces dernières dépassent largement le cadre de l'analyse de la stabilité et de l'interaction car les groupes peuvent être réalisés sur la base de la stabilité ou bien à partir de tout autre critère.

Le sélectionneur dispose d'outils intéressants et performants. Selon ses objectifs de sélection en matière de stabilité, objectifs qui dépendent de l'espèce, du type variétal, du type de milieu, du type de sélection (directe ou indirecte) mais aussi de ses moyens, il peut choisir la ou les méthodes les plus appropriées.
Cependant, nous pensons que de nouveaux progrès méthodologiques seront encore réalisés. Un indice en est donné par la production continuelle d'articles sur le sujet. En particulier, la prise en compte de la structure lieux (fixes) années (aléatoires), caractéristique du facteur environnement, conduit à l'analyse statistique de tableaux à trois entrées, une des voies les plus prometteuses.

\section{RÉFÉRENCES}

Abou-El-Fittouh HA, Rawlings JO, Miller PA (1969) Classification of environments to control genotype by environment interactions with an application to cotton. Crop Sci 9, 135-140

Allard RW, Bradshaw AD (1964) Implications of genotype- environmental interactions in plant breeding. Crop Sci 4, 503-508

Argillier O, Hébert Y, Barrière Y (1994) Statistical analysis and interpretation of line $X$ environment interaction for biomass yield in maize. agronomie 14, 661-672

Balfourier F, Charmet G (1991) Relationships between agronomic characters and ecogeographical factors in a collection of French perennial ryegrass populations. agronomie 11, 645-657

Baril CP (1992) Factor regression for interpreting genotype-environment interaction in bread wheat trials. Theor appl Genet 83, 1022-1026

Baril CP, Denis JB, Brabant P (1994) Selection of environments using simultaneous clustering based on genotype $\times$ environment interaction. Can J Plant Sci 74, 311-317

Baril CP, Denis JB, Wustman R, Van Eeuwijk FA (1995) Analysing genotype by environment interaction in Dutch potato variety trials using factorial regression. Euphytica 82, 149-155

Becker HC (1981) Correlations among some statistical measures of phenotypic stability. Euphytica 30 , 835-840

Becker HC, Léon J (1988) Stability analysis in plant breeding. Plant Breed 101, 1-23

Beckett JL (1982) Variety $\times$ environment interactions in sugar beet variety trials. J Agric Sci Camb 98, 425-435

Biarnès-Dumoulin V, Denis JB, Lejeune-Hénaut I, Etévé G (1996). Interpreting yield instability in pea using genotypic and environmental covariates. Crop Sci 36, 115-120

Bilbro JD, Ray LL (1976) Environmental stability and adaptation of several cotton cultivars. Crop Sci 16, 821-824

Borghi B, Perenzin M (1990) Yield and yield stability of conventional varieties and Fl bread wheat hybrids. J Genet Breed 44, 307-310 
Brancourt-Hulmel M, Lecomte C (1994) Sélection et stabilité du rendement chez le blé tendre d'hiver. agronomie 14, 611-625

Brandle JE, Arthur LM (1992) A comparative evaluation of two methods of selecting locations used for testing spring wheat cultivars. Theor Appl Genet 83, 301-304

Breese EL (1969) The measurement and significance of genotype-environment interactions in grasses Heredity 24, 27-44

Brown KD, Sorrells ME, Coffman WR (1983) Method for classification and evaluation of testing environments. Crop Sci 23, 889-893

Bull JK, Basford KE, Cooper M, DeLacy IH (1994) Enhanced interpretation of pattern analyses of environments: the use of blocks. Field Crops Res 37, 25-32

Bull JK, Cooper M, DeLacy IH, Basford KE, Woodruff DR (1992) Utility of repeated checks for hierarchical classification of data from plant breeding trials. Field Crops Res 30, 79-95

Byth DE, Eisemann RL, DeLacy IH (1976) Two-way pattern analysis of a large data set to evaluate genotypic adaptation. Heredity 37, 215-230

Campbell LG, Lafever HN (1977) Cultivar $\times$ environment interactions in soft red winter wheat yield tests. Crop Sci 17, 604-608

Ceccarelli S (1989) Wide adaptation : How wide? Euphytica 40, 197-205

Ceccarelli S, Grando S, Hamblin J (1992) Relationship between barley grain yield measured in low- and high-yielding environments. Euphytica 64, 49-58

Charmet G, Balfourier F, Ravel C, Denis JB (1993) Genotype $\times$ environment interactions in a core collection of French perennial ryegrass populations. Theor Appl Genet 86, 731-736

Cooper M, Byth DE, Woodruff DR (1994) An investigation of the grain yield adaptation of advanced CIMMYT wheat lines to water stess environments in Queensland. Il. Classification analysis. Aust $J$ Agric Res 45, 985-1002

Cornelius PL (1993) Statistical tests and retention of terms in the additive main effects and multiplicative interaction model for cultivar trials. Crop Sci 33, 1186-1193

Cornelius PL, Crossa J, Seyedsadr MS (1996) Statistical tests and estimators of multiplicative models for genotype-by-environment interaction. In: Genotype-by-Environment Interaction (Kang MS, Gauch HG, eds), CRC-Press, Boca Raton, 199234

Cornelius PL, Seyedsadr M, Crossa J (1992) Using the shifted multiplicative model to search for 'separability' in crop cultivar trials. Theor Appl Genet 84, $161-172$

Cornelius PL, Van Sanford DA, Seyedsadr MS (1993) Clustering cultivars into groups without rank-change interactions. Crop Sci 33,1193-1200
Corsten LCA, Denis JB (1990) Structuring interaction in two-way tables by clustering. Biometrics 46,207 215

Crossa J (1990) Statistical analyses of multilocation trials. Adv Agron 44, 55-85

Crossa J, Cornelius PL, Seyedsadr MS (1996) Using the shifted multiplicative model cluster methods for crossover stability. In : Genotype-by-Environment Interaction (Kang MS, Gauch HG, eds), CRCPress, Boca Raton, 175-198

Crossa J, Cornelius PL, Seyedsadr MS, Byrne P (1993) A shifted multiplicative model cluster analysis for grouping environments without genotypic range change. Theor Appl Genet 85, 577-586

Crossa J, Fox PN, Pfeiffer WH, Rajaram S, Gauch HG (1991) AMMI adjustment for statistical analysis of an international wheat yield trial. Theor Appl Genet $81,27-37$

Cruz RM (1992) More about the multiplicative model for the analysis of genotype-environment interaction. Heredity $68,135-140$

Decoux G, Denis JB (1991) Intera. Logiciels pour l'interprétation statistique de l'interaction entre deux facteurs. Laboratoire de biométrie, Inra, route de Saint-Cyr, 78026 Versailles cedex, France

Denis JB (1979) Structuration de l'interaction. Biom Praxim 19, 15-34

Denis JB (1980) Analyse de régression factorielle. Biom Praxim 20, 1-34

Denis JB (1988) Two way analysis using covariates. Statistics 19, 123-132

Denis JB (1992) Analyse statistique de l'interaction entre deux facteurs. Support de cours, laboratoire de biométrie, Inra, route de Saint-Cyr, 78026 Versailles cedex, France

Denis JB, Baril CP (1992) The multiplicative interaction model with numerous missing values. Biuletyn Oceny Odmina 24, 33-45

Denis JB, Gower JC (1992) Biadditive models. Technical report, laboratoire de biométrie, Inra, route de Saint-Cyr, 78026 Versailles cedex, France

Denis JB, Gower JC (1996) Asymptotic confidence regions for biadditive models: interpreting genotype-environment interactions. Appl Stat 4, 479-493

Denis JB, Piepho HP, van Eeuwijk FA (1997) Modelling expectation and variance for genotype by environment data. Heredity (sous presse)

Denis JB, Vincourt P (1982) Panorama des méthodes statistiques d'analyse des interactions génotype $X$ milieu. agronomie 2, 219-230

Dumoulin V 1994. Étude de la variabilité génétique chez le pois protéagineux (Pisum sativum L.) pour l'élaboration du rendement. Importance des interactions génotype $\times$ milieu. Thèse de l'Ina-PG, Ina-PG, Paris

Eagles HA, Frey KJ (1977) Repeatability of the stability-variance parameter in oats. Crop Sci 17, 253-256 
Eberhart SA, Russell WA (1966) Stability parameters for comparing varieties. Crop Sci 6, 36-40

Eberhart SA, Russell WA (1969) Yield and stability for a 10-line diallel of single-cross and double-cross maize hybrids. Crop Sci 9 , 357-361

Eskridge KM, Mumm RF (1992) Choosing plant cultivars based on the probability of outperforming a check. Theor Appl Genet 84, 494-500

Eskridge KM, Smith OS, Byrne PF (1993) Comparing test cultivars using reliability functions of testcheck differences from on-farm trials. Theor Appl Genet 87, 60-64

Feyerherm AM, Sears RG, Paulsen GM (1992) Utilizing genotype-by-weather interactions for selecting wheat cultivars. Agron J 84, 391-394

Finlay KW, Wilkinson GN (1963) The analysis of adaptation in a plant-breeding programme. Aust $J$ Agric Res 14, 742-754

Fox PN, Rosielle AA (1982) Reducing the influence of environmental main-effects on pattern analysis of plant breeding environments. Euphytica 31, 645656

Fox PN, Skovmand B, Thompson BK, Braun HJ, Cormier R (1990) Yield and adaptation of hexaploid spring triticale. Euphytica 47, 57-64

Francis TR, Kannenberg LW (1978) Yield stability studies in short-season maize. I. A descriptive method for grouping genotypes. Can J Plant Sci 58, 1029-1034

Freeman GH (1973) Statistical methods for the analysis of genotype-environment interactions. Heredity $31,339-354$

Freeman GH (1990) Modern statistical methods for analyzing genotype $\times$ environment interactions. In: Genotype-by-Environment Interaction and Plant Breeding (Kang MS, ed), LSU Agricultural Center, Baton Rouge, 118-125

Freeman GH, Perkins JM (1971) Environmental and genotype-environmental components of variabillity. VIII Relations between genotypes grown in different environments and measures of these environments. Heredity 27, 15-23

Gallais A (1992a) Adaptation et adaptabilité en amélioration des plantes. Sel FR 42, 55-57

Gallais A (1992b) Bases génétiques et stratégie de sélection de l'adaptation générale. Sel FR 42, 59-78

Gauch HG (1990) Full and reduced models for yield trials. Theor Appl Genet 80, 153-160

Gauch HG (1992) Statistical analysis of regional yield trials: AMMI analysis of factorial designs. Elsevier, Amsterdam

Gauch HG, Zobel RW (1988) Predictive and postdictive success of statistical analyses of yield trials. Theor Appl Genet 76, 1-10

Gauch HG, Zobel RW (1996) AMMI analysis of yield trials. In : Genotype-by-Environment Interaction (Kang MS, Gauch HG, eds), CRC Press, Boca Raton, 85-122
Ghaderi A, Adams MW, Saettler AW (1982) Environmental response patterns in commercial classes of common bean (Phaseolus vulgaris L). Theor Appl Genet 63, 17-22

Ghaderi A, Everson EH, Cress CE (1980) Classification of environments and genotypes in wheat. Crop Sci 20, 707-710

Giauffret C, Bonhomme R, Derieux M (1997) Heterosis in maize for biomass production, leaf area establishment, and radiation use efficiency under cool spring conditions. Maydica 42, 13-19

Gollob HF (1968) A statistical model which combines features of factor analytic and analysis of variance techniques. Psychom 33, 73-115

Gorman DP, Kang MS, Milam MR (1989) Contribution of weather variables to genotype $x$ environment interaction in grain sorghum. Plant Breed 103, 299-303

Gray E (1982) Genotype-environment interactions and stability analysis for forage yield of orchardgrass clones. Crop Sci 22, 19-23

Guénoche A, Vandeputte-Riboud B, Denis JB (1994) Selecting varieties using a series of trials and a combinatorial ordering method. agronomie 14, 363375

Gusmao L, Mexia JT, Baeta J (1992) Trimmed joint regression: a new approach to the joint regression analysis for cultivar relative-performance evaluation. Theor Appl Genet 84, 735-738

Habgood RM (1977) Estimation of genetic diversity of self-fertilizing cereal cultivars based on genotypeenvironment interactions. Euphytica 26, 485-489

Hanson WD (1970) Genotypic stability. Theor Appl Genet 40, 226-231

Hardwick RC, Wood JT (1972) Regression methods for studying genotype-environment interactions. Heredity 28, 209-222

Haussmann BIG, Geiger HH (1994) Effects of heterozygosity and heterogeneity on the adaptation of sorghum (Sorghum bicolor (L) Moench) to a semiarid area of Kenya. In : Biometrics in Plant Breeding: Applications of Molecular Markers (van Ooijen JW, Jansen J, eds), Proceedings of the ninth meeting of the EUCARPIA Section Biometrics in Plant Breeding, Wageningen, the Netherlands, 6-8. July 1994, 107-115

Helms TC (1993) Selection for yield and stability among oat lines. Crop Sci 33, 423-426

Hoogendoorn J, Pfeiffer WH, Rajaram S, Gale MD (1988) Adaptive aspects of dwarfing genes in CIMMYT germplasm. Proceedings of the seventh international wheat genetics symposium, held at Cambridge, UK, 13-19 july 1988, $1093-1100$

Hühn M (1990a) Nonparametric measures of phenotypic stability. Part 1: Theory. Euphytica 47, 189-194

Hühn M (1990b) Nonparametric measures of phenotypic stability. Part 2: Applications. Euphytica 47,195-201 
Hühn M (1996) Nonparametric analysis of genotype*environments by ranks. In : Genotype-byEnvironment Interaction (Kang MS, Gauch HG, eds), CRC Press, Boca Raton, 235-271

Hühn M, Lolito S, Piepho HP (1993) Relationships between genotype $\times$ environment interactions and rank orders for a set of genotypes tested in different environments. Theor Appl Genet 86, 943-950

Imrie BC, Drake DW, De Lacy IH, Byth DE (1981) Analysis of genotypic and environmental variation in international mungbean trials. Euphytica 30, 301311

Jackson PA, Byth DE, Fischer KS, Johnston RP (1994) Genotype $\times$ environment interactions in progeny from a barley cross. II. Variation in grain yield, yield components and dry matter production among lines with similar times to anthesis. Field Crops Res 37, 11-23

Jackson PA, Byth DE, Johnston RP, Fischer KS (1993) Genotype $\times$ environment interactions in progeny from a barley cross. 1. Patterns of response among progeny lines for grain yield and time to anthesis. Aust J Exp Agric 33, 619-627

Jalaluddin MD, Harrison SA (1993) Repeatability of stability estimators for grain yield in wheat. Crop Sci 33, 720-725

Johnson GR (1977) Analysis of genotypic similarity in terms of mean yield and stability of environmental response in a set of maize hybrids. Crop Sci 17 , $837-842$

Jones HG (1979) Effects of weather on spring barley yields in Britain. J Natn Inst Agric Bot 15, 24-33

Kang MS (1988) A rank-sum method for selecting high yielding, stable corn genotypes. Cereal Res Commun 16,113-115

Kang MS, Gauch HG, eds (1996) Genotype-byEnvironment Interaction (Kang MS, Gauch $\mathrm{HG}$, eds), CRC-Press, Boca Raton

Kang MS, Gorman DP (1989) Genotype $\times$ environment interaction in maize. Agron J 81, 662-664

Kang MS, Pham HN (1991) Simultaneous selection for high yielding and stable genotypes. Agron $J 83$, 161-165

Kearsey MJ, Hayward MD, Devey FD, Arcioni S, Eggleston MP, Eissa MM (1987) Genetic analysis of production characters in Lolium. 1. Triple test cross analysis of spaced plant performance. Theor Appl Genet 75, 66-75

Kempton RA (1984) The use of biplots in interpreting variety by environment interactions. J Agric Sci $103,123-135$

Krenzer EG, Thompson JD, Carver BF (1992) Partitioning of genotype $x$ environment interactions of winter wheat forage yield. Crop Sci 32, 1143 1147

Lefkovitch LP (1985) Multi-criteria clustering in genotype-environment interaction problem. Theor Appl Genet 70, 585-589
Lefort-Buson M, Dattée Y (1985) Étude de l'hérérosis chez le colza oléagineux d'hiver (Brassica napus L.) I. - Comparaison de deux populations, l'une homozygote et l'autre hétérozygote. agronomie 5, 101-110

Léon J, Becker HC (1988) Repeatability of some statistical measures of phenotypic stabilityCorrelations between single year results and multi years results. Plant Breed 100, 137-142

Lin CS (1982) Grouping genotypes by a cluster method directly related to genotype-environment interaction mean square. Theor Appl Genet 62, 277280

Lin CS, Binns MR (1988a) A method of analyzing cultivar $\times$ location $\times$ year experiments: a new stability parameter. Theor Appl Genet 76, 425-430

Lin CS, Binns MR (1988b) A superiority measure of cultivar performance for cultivar * location data. Can J Plant Sci 68, 193-198

Lin CS, Binns MR (1991) Assessment of a method for cultivar selection based on regional trial data. Theor Appl Genet 82, 379-388

Lin CS, Binns MR, Lefkovitch LP (1986) Stability analysis: where do we stand? Crop Sci 26, 894-900

Lin CS, Butler G (1988) A data-based approach for selecting locations for regional trials. Can J Plant Sci 68, 651-659

Lin CS, Butler G (1990) Cluster analyses for analyzing two-way classification data. Agron J 82, 344-348

Lin CS, Butler G, Hall I, Nault C (1992) Program for Investigating genotype-environment interaction. Agron J 84, 121-124

Lin CS, Morrison MJ (1992) Selection of test locations for regional trials of barley. Theor Appl Genet 83, 968-972

Lin CS, Thompson B (1975) An empirical method of grouping genotypes based on a linear function of the genotype-environment interaction. Heredity 34 , 255-263

Mandel J (1969) The partitioning of interaction in analysis of variance. Journal of Research of the National Bureau of Standards B. Mathematical Sciences 73B, 309-328

Mandel J (1971) A new analysis of variance model for non-additive data. Technometrics 13, 1-18

Milliken GA, Graybill FA (1970) Extensions of the general linear model. J Am Stat Assoc 65, 797-807

Mungomery VE, Shorter R, Byth DE (1974) Genotype $\times$ environment interactions and environmental adaptation. I pattern analysis-application to soya bean populations. Aust J Agric Res 25, 59-72

Nachit MM, Sorrells ME, Zobel RW, Gauch HG, Fischer RA, Coffman WR (1992a) Association of morpho-physiological traits with grain yield and components of genotype-environment interaction in durum wheat. I $J$ Genet Breed 46, 363-368

Nachit MM, Sorrells ME, Zobel RW, Gauch HG, Fischer RA, Coffman WR (1992b) Association of 
environmental variables with sites' mean grain yield and components of genotype-environment interaction in durum wheat. II $J$ Genet Breed 46, 369-372

Nguyen HT, Sleper DA, Hunt KL (1980) Genotype environment interactions and stability analysis for herbage yield of tall fescue synthetics. Crop Sci 20 , 221-224

Nor KM, Cady FB (1979) Methodology for identifying wide adaptability in crops. Agron J 71, 556-559

Oman SD (1991) Multiplicative effects in mixed model analysis of variance. Biometrika 78, 729-739

Ombakho GA, Miller FR (1994) Performance, stability, and prediction of performance in single, threeway, and double-cross hybrids of sorghum. Int Sorghum Millets Newsl 35, 77

Parisot-Baril C (1992) Étude de la stabilité du rendement chez le blé tendre d'hiver (Triticum aestivum L. Thell.) Thèse de doctorat de l'université ParisSud

Perkins JM (1972) The principal component analysis of genotype-environmental interactions and physical measures of the environment. Heredity 32, 5170

Perkins JM, Jinks JL (1968a) Environmental and genotype-environmental components of variability. III. Multiple lines and crosses. Heredity 23, 339-356

Perkins JM, Jinks JL (1968b) Environmental and genotype-environmental components of variability. IV. Non-linear interactions for multiple inbred lines. Heredity 23, 525-535

Peterson CJ (1992) Similarities among test sites based on cultivar performance in the hard red winter wheat region. Crop Sci 32, 907-912

Pham HN, Kang MS (1988) Interrelationships among and repeatability of several stability statistics estimated from international maize trials. Crop Sci 28 , 925-928

Piepho HP, Lolito S (1992) Rank correlation among parametric and nonparametric measures of phenotypic stability. Euphytica 64, 221-225

Pinthus MJ (1973) Estimate of genotypic value: a proposed method. Euphytica 22, 121-123

Rameau C, Denis JB (1992) Characterization of environments in long-term multi-site trials in Asparagus, through yield of standard varieties and use of environmental covariates. Plant Breed 109 , 183-191

Ramey TB, Rosielle AA (1983) HASS cluster analysis: a new method of grouping genotypes or environments in plant breeding. Theor Appl Genet 66, $131-133$

Romagosa I, Fox PN (1993) Genotype x environment interaction and adaptation. In: Plant Breeding: Principles and Prospects. (Hayward MD, Bosemark NO, Romagosa I, eds), Chapman \& Hall, Londres, 373-390
Romagosa I, Fox PN, Garcia del Moral LF, Ramos JM, Garcia del Moral B, Roca de Togores F, Molina-Cano JL (1993) Integration of statistical and physiological analyses of adaptation of near-isogenic barley lines. Theor appl Genet 86, 822-826

Saeed M, Francis CA (1984) Association of weather variables with genotype $\times$ environment interactions in grain sorghum. Crop Sci 24, 13-16

Saindon G, Schaalje GB (1993) Evaluation of locations for testing dry bean cultivars in western Canada using statistical procedures, biological interpretation and multiple traits. Can J Plant Sci $73,985-994$

Sarawat P, Stoddard FL, Marshall DR, Ali SM (1994) Hererosis for yield and related characters in pea. Euphytica 80, 39-48

Scheffé H (1959) The analysis of variance. John Wiley, New York

Seyedsadr M, Cornelius PL (1992) Shifted multiplicative models for nonadditive two-tables. Commun Stat Simul 21, 807-832

Shafii B, Mahler KA, Price WJ, Auld DL (1992) Genotype $x$ environment interaction effects on winter rapeseed yield and oil content. Crop Sci 32, 922927

Shorter R, Byth DE, Mungomery VE (1977) Genotype $x$ environment interactions and environmental adaptation. II. Assessment of environmental contributions. Aust J Agric Res 28, 223-235

Shukla GK (1972) Some statistical aspects of partitioning genotype-environmental components of variability. Heredity 29, 237-245

Simane B, Struik PC, Nachit MM, Peacock JM (1993) Ontogenetic analysis of yield components and yield stability of durum wheat in water-limited environments. Euphytica 71, 211-219

Stelling D, Ebmeyer E, Link W (1994) Yield stability in Faba bean, Vicia faba L. 2. Effects of heterozygosity and heterogeneity. Plant Breed 112, 30-39

Tai GCC (1975) Analysis of genotype-environment interactions based on the method of path coefficient analysis. Can J Genet Cytol 17, 141-149

Tai GCC (1990) Path analysis of genotype--environment interactions. In: Genotype-by-Environment Interaction and Plant Breeding (Kang MS, ed), LSU Agricultural Center, Baton Rouge, 273-286

Tai GCC, Levy D, Coleman WK (1994) Path analysis of genotype-environment interactions of potatoes exposed to increasing warm-climate constraints. Euphytica 75, 49-61

Tan WK, Tan GY, Walton PD (1979) Regression analysis of genotype-environment interaction in smooth bromegrass. Crop Sci 19, 393-396

van Eeuwijk FA (1992) Interpreting genotype-by-environment interaction using redundancy analysis. Theor Appl Genet 85, 89- 100

van Eeuwijk FA (1996) Between and beyond additivity and non-additivity / the statistical modelling of 
genotype by environment interaction in plant breeding. PhD Thesis University of Wageningen, Wageningen

van Eeuwijk FA, Denis JB, Kang MS (1996) Incorporating additional information on genotypes and environments in models for two-way genotype by environment tables. In : Genotype-byEnvironment Interaction (Kang MS, Gauch $\mathrm{HG}$, eds), CRC-Press, Boca Raton, 15-49

van Eeuwijk FA, Elgersma A (1993) Incorporating environmental information in an analysis of genotype by environment interaction for seed yield in perennial ryegrass. Heredity 70, 447-457

Verma MM, Chahal GS, Murty BR (1978) Limitations of conventional regression analysis. A proposed modification. Theor Appl Genet 53, 89-91

Vincourt P, Derieux M, Gallais A (1984) Quelques méthodes de choix des génotypes à partir d'essais multilocaux. agronomie 4, 843-848

von Wricke G (1962) Über eine Methode zur Erfassung der ökologischen Streubreite in Feldversuchen. Z Planzenzücht 47, 92-96

Weatherup STC (1994) Use of Mahalanobis distance to measure varietal distinctness. Plant Var Seeds 7 , 107-119
Westcott B (1986) Some methods of analysing genotype-environment interactions. Heredity 56, 243-253

Westcott B (1987) A method of assessing the yield stability of crop genotypes. J agric Sci Camb 108, 267-274

Wood JT (1976) The use of environmental variables in the interpretation of genotype-environment interaction. Heredity 37, 1-7

Worland AJ (1996) The influence of flowering time genes on environmental adaptability in European wheats. Euphytica 89, 49-57

Wright AJ (1971) The analysis and prediction of some two factor interactions in grass breeding. J Agric Sci Camb 76, 301-306

Yates F, Cochran WG (1938) The analysis of groups of experiments. $J$ Agric Sci $28,556-580$

Yau SK, Ortiz-Ferrara G, Srivastava JP (1991) Classification of diverse bread wheat-growing environments based on differential yield responses. Crop Sci 31, 571-576

Zobel RW, Wright MW, Gauch HG (1988) Statistical analysis of a yield trial. Agron J 80, 388-393 